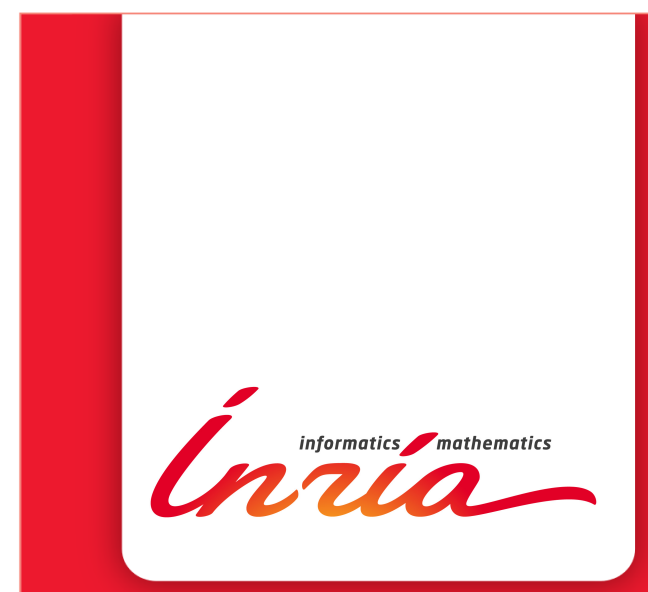

Equilibrium reconstruction at JET using

Stokes model for polarimetry

Blaise Faugeras, Francesco Orsitto

RESEARCH 



\title{
inzía
}

\section{Equilibrium reconstruction at JET using Stokes model for polarimetry}

\author{
Blaise Faugeras, Francesco Orsitto \\ Project-Team CASTOR \\ Research Report n 9153 - February 2018 - 29 pages
}

\begin{abstract}
This paper presents the first application to real JET data of the new equilibrium code NICE which enables the consistent resolution of the inverse equilibrium reconstruction problem in the framework of non-linear free-boundary equilibrium coupled to the Stokes model equation for polarimetry. The conducted numerical experiments enable first of all to validate NICE by comparing it to the well-established EFIT code on 4 selected high performance shots. Secondly the results indicate that the fit to polarimetry measurements clearly benefits from the use of Stokes vector measurements compared to the classical case of Faraday measurements, and that the reconstructed profiles are better constrained with smaller error bars and are closer from the profiles reconstructed by EFTM, the EFIT JET code using internal MSE constraints.
\end{abstract}

Key-words: Tokamak equilibrium reconstruction, Stokes model polarimetry, inverse problem, PDE-constrained optimization, JET

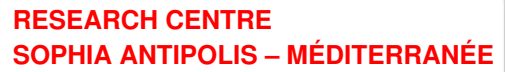


Résumé : Ce travail présente la première utilisation de données réelles du tokamak JET avec le code NICE pour la reconstruction de l'équilibre d'un plasma à frontière libre et utilisant le modèle de Stokes pour la polarimétrie.

Mots-clés : Reconstruction d'équilibre, tokamak, polarimètrie, modèle de Stokes, problème inverse, EDP, optimisation, JET 


\section{Introduction}

Numerical equilibrium reconstruction is an important and long standing subject in tokamak fusion plasma science [28, 31, 17, 30, 14, 22. The resolution of this inverse problem: the reconstruction of the poloidal flux function and of the plasma boundary as well as the identification of two non-linear source term functions known as $p^{\prime}$ and $f f^{\prime}$ in the Grad-Shafranov equation [10, 29, 16, is needed on the one hand for real time control of the plasma during a discharge and on the other hand for post-treatment analysis of equilibrium configurations. The basic set of measurements needed and used are magnetic probes and flux loops which provide values of the poloidal magnetic field and flux at several points surrounding the vacuum vessel and the plasma. All free boundary reconstruction codes (e.g. [15, 6, 18, 32, 3, 4, 8, 20]) primarily use these magnetic measurements which proved to be sufficient to identify correctly the plasma boundary and the averaged plasma current density profile [4. However the difficulty of the reconstruction of the current profile, when only magnetic measurements are used, has been pointed out in [26] and is inherent to the ill-posedness of this inverse problem.

In order to be able to separate more precisely the contributions of the two non-linearities $p^{\prime}$ and $f f^{\prime}$ it appears necessary to use supplementary measurements. Interferometry and polarimetry provide integrated quantities along lines of sight or chords crossing the plasma poloidal section [13, 15, 6, 5, 4. Even though less informative than motional Stark effect (MSE) measurements, polarimetry has the great advantage of being routineley available during a shot. Equilibrium reconstruction codes have until now use Faraday rotation angle measurements only. Moreover the modelization used to represent these Faraday rotation measurements is known to be a too rough approximation for ITER plasma regimes where the Cotton-Mouton effects is expected to be much stronger than in today's tokamaks as a result of high plasma currents and high electron density. The relativistic effect is also expected to be stronger as a result of the high electron temperature 19 .

In [25, 23, 24, it has been shown using JET measurements that for high plasma currents and electron density the polarimetry Faraday effect cannot be considered alone independently from the Cotton-Mouton effect and that the coupling between them has to be taken into account. So to do the Stokes model for polarimety [27] has to be used instead of its approximation commonly used in equilibrium reconstruction codes.

In a recent paper [9] a numerical method enabling the consistent resolution of the inverse equilibrium reconstruction problem in the framework of non-linear free-boundary equilibrium coupled to the Stokes model equation, was proposed and validated using synthetic ITER measurements.

In the present work we use for the first time this method in order to perform equilibrium reconstruction at JET using real Stokes vector measurements. This has demanded the development of a new equilibrium code called NICE (Newton direct and Inverse Computation for Equilibrium).

Next Section 2 is devoted to the formulation of the direct model and the inverse equilibirum reconstruction problem at JET. It ends with a brief description of the numerical methods implemented in NICE. Section 3 presents numerical results using NICE with real JET data. Verification and validation are done by comparison with the well-established EFIT, EFTF and EFTM codes at JET. 


\section{Inverse problem formulation}

\subsection{Free-boundary plasma equilibrium}

Assuming axial symmetry and introducing a cylindrical coordinate system $\left(\boldsymbol{e}_{r}, \boldsymbol{e}_{\phi}, \boldsymbol{e}_{z}\right)$ we consider the classical non-linear free-boundary Grad-Shafranov equilibrium model for the poloidal flux $\psi(r, z)$

$$
\begin{cases}-\Delta^{*} \psi=\left[\lambda\left(\frac{r}{r_{0}} \mathcal{A}\left(\psi_{\mathrm{N}}\right)+\frac{r_{0}}{r} \mathcal{B}\left(\psi_{\mathrm{N}}\right)\right)\right] 1_{\Omega_{\mathrm{p}}(\psi)} & \text { in } \Omega \\ \psi=g & \text { on } \partial \Omega\end{cases}
$$

Here $\Delta^{*}$ is defined by

$$
\Delta^{*} .:=\nabla \cdot\left(\frac{1}{\mu_{0} r} \nabla .\right) .
$$

$\nabla$ is the $2 \mathrm{D}$ operator in the $(r, z)$-plane and $\mu_{0}$ is the magnetic permeability of vacuum.

The fixed bounded computational domain $\Omega$ contains (or might be equal to) the limiter domain $\Omega_{\mathrm{L}}\left(\partial \Omega_{\mathrm{L}}\right.$ is the limiter contour) accessible to the plasma. Cauchy conditions $(g, h):=$ $\left(\psi, \frac{1}{r} \partial_{n} \psi\right)$ are assumed to be given on the domain boundary or measurement contour $\partial \Omega$. This can be achieved by interpolation and extrapolation of the different magnetic measurements [22, 8 ]

The right hand side of Eq. (1) represents the toroidal component of the current density in the plasma, $j_{\phi}=r p^{\prime}(\psi)+\frac{1}{r \mu_{0}} f f^{\prime}(\psi)$, expressed using the adimentionalized functions $\mathcal{A}$ and $\mathcal{B}$, the major radius $r_{0}$ and a scaling factor $\lambda .1_{\Omega_{\mathrm{p}}(\psi)}$ is the indicator function of the unknown plasma domain. This domain is defined by its boundary which is the outermost closed $\psi$ iso-contour contained within the limiter domain $\Omega_{\mathrm{L}}$. The plasma can either be limited if this iso-contour is tangent to the limiter $\partial \Omega_{\mathrm{L}}$ or defined by the presence of an X-point.

The normalized poloidal flux $\psi_{\mathrm{N}}(r, z)$ is

$$
\psi_{\mathrm{N}}(r, z)=\frac{\psi(r, z)-\psi_{\mathrm{a}}(\psi)}{\psi_{\mathrm{b}}(\psi)-\psi_{\mathrm{a}}(\psi)},
$$

with $\psi_{\mathrm{a}}$ and $\psi_{\mathrm{b}}$ being the flux values at the magnetic axis and at the boundary of the plasma:

$$
\begin{aligned}
& \psi_{\mathrm{a}}(\psi):=\psi\left(r_{\mathrm{a}}(\psi), z_{\mathrm{a}}(\psi)\right), \\
& \psi_{\mathrm{b}}(\psi):=\psi\left(r_{\mathrm{b}}(\psi), z_{\mathrm{b}}(\psi)\right),
\end{aligned}
$$

with $\left(r_{\mathrm{a}}(\psi), z_{\mathrm{a}}(\psi)\right)$ the magnetic axis, where $\psi$ has its global maximum in $\Omega_{\mathrm{L}}$ and $\left(r_{\mathrm{b}}(\psi), z_{\mathrm{b}}(\psi)\right)$ the coordinates of the point which determines the plasma boundary. The point $\left(r_{\mathrm{b}}, z_{\mathrm{b}}\right)$ is either an $\mathrm{X}$-point of $\psi$ or the contact point with the limiter $\partial \Omega_{\mathrm{L}}$.

\subsection{Stokes model for polarimetry}

Polarimetry consists in measurements of the change of state of polarization of an electromagnetic radiation propagating across the magnetized plasma along several lines of sight distributed on the poloidal section of the tokamak. One method of describing the state of polarization is to introduce a Stokes vector $\mathbf{s}=\left(s_{1}, s_{2}, s_{3}\right)$. The evolution of the polarization when the laser beam crosses the plasma is then given by the following Stokes equation on each line of sight:

$$
\left\{\begin{array}{l}
\frac{d \mathbf{s}}{d Z}=\mathbf{G s}, \quad \text { on }\left(Z_{0}, Z_{1}\right] \\
\mathbf{s}\left(Z_{0}\right)=\mathbf{s}_{0}
\end{array}\right.
$$


We refer to [27] for details on this modelization. Here we have introduced a coordinate system $\left(e_{X}, e_{Y}, e_{Z}\right)$ attached to a line of sight $L . Z$ is the coordinate tangent to the chord, $X$ represents the toroidal direction and $Y$ the direction perpendicular to $Z$ in the poloidal plane. In this coordinate system the components of the magnetic field are denoted by $\left(B_{X}, B_{Y}, B_{Z}\right)$.

The initial polarization is given by $\mathbf{s}_{0}$ at $Z_{0}$. $Z_{1}$ corresponds to the location of the output measurement sensor. The $3 \times 3$ matrix $\boldsymbol{G}$ is such that $\boldsymbol{G s}=\boldsymbol{\Omega} \times \mathbf{s}$ where vector $\boldsymbol{\Omega}=\left(\Omega_{1}, \Omega_{2}, \Omega_{3}\right)$ has components

$$
\Omega_{1}=c_{1} N_{e}\left(B_{X}^{2}-B_{Y}^{2}\right), \quad \Omega_{2}=2 c_{1} N_{e} B_{X} B_{Y}, \quad \Omega_{3}=c_{3} N_{e} B_{Z} .
$$

Here the electron density in the plasma, $N_{e}=N_{e}\left(\psi_{\mathrm{N}}\right)$, is assumed to be constant on the flux surfaces. The components of the magnetic field can be written as

$$
B_{Z}=-\frac{1}{r} \nabla \psi \cdot e_{Y}, \quad B_{Y}=\frac{1}{r} \nabla \psi \cdot e_{Z}, \quad B_{X}=\frac{f}{r}
$$

where the diamagnetic function $f$ is related to function $\mathcal{B}$ from Eq. (1) through the relation $f f^{\prime}=\lambda \mu_{0} r_{0} \mathcal{B}$. In order to keep notations consistant let us also introduce a normalizing constant $\lambda_{e}$ and a function $\mathcal{C}$ such that $N_{e}\left(\psi_{\mathrm{N}}\right)=\lambda_{e} \mathcal{C}\left(\psi_{\mathrm{N}}\right)$. The dependence of $\boldsymbol{G}$ on $\psi$, on the electron density function $\mathcal{C}$ and on function $\mathcal{B}$ is denoted by $\boldsymbol{G}(\psi, \mathcal{B}, \mathcal{C})$. Constants $c_{1}$ and $c_{3}$ depend on the wavelength of the beam radiation.

Hence in order to use polarimetry measurements with Stokes modelization for the equilibrium reconstruction problem one has to supplement equation (1) with, for each line of sight, a system of linear ordinary differential equations (5) for the Stokes vector.

\subsection{The inverse identification problem}

Magnetics constitute the basic set of experimental measurements used in equilibrium reconstruction for the identification of functions $\mathcal{A}$ and $\mathcal{B}$. As already mentioned earlier, in this study, magnetics are preprocessed and we consider that Cauchy condition $(g, h)=\left(\psi, \frac{1}{r} \partial_{n} \psi\right)$ are given on $\partial \Omega$. Dirichlet conditions $g$ are used in the direct model (1) and Neumann conditions $h$ are used in the inverse problem cost function.

In order to be able to use polarimetric measurements the electron density function, $\mathcal{C}\left(\psi_{\mathrm{N}}\right)$ has to be known. It is therefore also going to be identified using interferometric measurements which give the density line integrals over each of the $N_{L}$ lines of sight $L^{i}, i=1, \ldots N_{L}$ :

$$
N_{e, o b s}^{i} \approx \int_{L^{i}} \lambda_{e} \mathcal{C}\left(\psi_{\mathrm{N}}\right) d Z^{i}
$$

A polarimetric measurement as it is considered in former equilibrium reconstruction studies (e.g. [13, 5, 4]) is the variatation of the Faraday rotation angle of the infrared radiation crossing the section of the plasma along a line of sight $L^{i}$ and is numerically evaluated by

$$
\delta \alpha_{o b s}^{i} \approx \frac{1}{2} W_{3}^{i} .
$$

Here we have introduced the notation

$$
W_{k}^{i}=\frac{1}{2} \int_{L^{i}} \Omega_{k} d Z^{i}
$$

As detailed in [27] Eq. (8) is an approximation to one component of the Stokes vector $\mathbf{s}\left(Z_{1}^{i}\right)$ only valid for small Faraday and Cotton-Mouton effects. On the contrary in this study we consider 
that polarimetric measurements are given by the full Stokes vector at the $Z_{1}^{i}$ coordinate on each chord $L^{i}$

$$
\mathbf{s}_{o b s}^{i} \approx \mathbf{s}\left(Z_{1}^{i}\right) .
$$

In order to use the same data as the JET codes EFTF and EFTM we also consider as measurements, pressure values, $p_{\text {obs }}^{i}$, provided at given points $x^{i}$. These values are to be compared to the ones computed by the integration of $p^{\prime}=\lambda \frac{1}{r_{0}} \mathcal{A}$ denoted below by $p_{\mathcal{A}}\left(x^{i}\right)$.

At this point we have defined a direct model given by the equilibrium equation (1) and Stokes equation (5) on every line of sight, control variables $\mathcal{A}, \mathcal{B}$ and $\mathcal{C}$, and measurements to which are attached experimental errors represented by the standard deviations $\sigma \mathrm{s}$ in Eq. (12) below. The identification problem can now be formulated as a constrained minimization problem for the following cost function $\left(\{\mathbf{s}\}\right.$ denotes the vector $\left(\mathbf{s}^{1}, \ldots, \mathbf{s}^{N_{L}}\right)$ of 3 -dimensional Stokes vectors for all lines of sight):

$$
J_{S}(\psi,\{\mathbf{s}\}, \mathcal{A}, \mathcal{B}, \mathcal{C}):=J_{o S}(\psi,\{\mathbf{s}\}, \mathcal{A}, \mathcal{C})+R(\mathcal{A}, \mathcal{B})+R_{C}(\mathcal{C}),
$$

with

$$
J_{o S}(\psi,\{\mathbf{s}\}, \mathcal{A}, \mathcal{C}):=J_{\text {mag }}(\psi)+J_{\text {interf }}(\psi, \mathcal{C})+J_{\text {polar } S}(\{\mathbf{s}\})+J_{\text {press }}(\psi, \mathcal{A})
$$

where the least-square misfit terms are

$$
\begin{aligned}
& J_{\text {mag }}(\psi):=\frac{1}{2 \sigma_{\text {mag }}^{2}} \int_{\partial \Omega}\left(\frac{1}{r} \partial_{n} \psi-h\right)^{2} d l \\
& J_{\text {interf }}(\psi, \mathcal{C}):=\sum_{i=1}^{N_{L}} \frac{1}{2 \sigma_{N_{i}}^{2}}\left(\int_{L_{i}} \lambda_{e} \mathcal{C}\left(\psi_{\mathrm{N}}\right) d Z-N_{e, o b s}^{i}\right)^{2}, \\
& J_{\text {polar } S}(\{\mathbf{s}\}):=\sum_{i=1}^{N_{L}} \frac{1}{2 \sigma_{S i}^{2}}\left\|\mathbf{s}^{i}\left(Z_{1}^{i}\right)-\mathbf{s}_{o b s}^{i}\right\|^{2} \\
& J_{\text {press }}(\psi, \mathcal{A}):=\sum_{i=1}^{N_{p}} \frac{1}{2 \sigma_{p i}^{2}}\left|p_{\mathcal{A}}\left(x^{i}\right)-p_{o b s}^{i}\right|^{2}
\end{aligned}
$$

and the regularization terms are

$$
\begin{aligned}
& R(\mathcal{A}, \mathcal{B})=\frac{\varepsilon_{A}}{2} \int_{0}^{1}\left[\mathcal{A}^{\prime \prime}(x)\right]^{2} d x+\frac{\varepsilon_{B}}{2} \int_{0}^{1}\left[\mathcal{B}^{\prime \prime}(x)\right]^{2} d x \\
& R_{C}(\mathcal{C})=\frac{\varepsilon_{C}}{2} \int_{0}^{1}\left[\mathcal{C}^{\prime \prime}(x)\right]^{2} d x
\end{aligned}
$$

The equilibrium reconstruction problem using Stokes model for polarimetry is formulated as:

Reconstruction problem 1 (with Stokes model) Find $(\psi,\{\mathbf{s}\}, \mathcal{A}, \mathcal{B}, \mathcal{C})$ minimizing $J_{S}(\psi,\{\mathbf{s}\}, \mathcal{A}, \mathcal{B}, \mathcal{C})$ from Eq. (10) under the constraint of the model equations (14) and (15) below:

$$
\begin{cases}-\Delta^{*} \psi=\left[\lambda\left(\frac{r}{r_{0}} \mathcal{A}\left(\psi_{\mathrm{N}}\right)+\frac{r_{0}}{r} \mathcal{B}\left(\psi_{\mathrm{N}}\right)\right)\right] 1_{\Omega_{\mathrm{p}}(\psi)} & \text { in } \Omega \\ \psi=g & \text { on } \partial \Omega\end{cases}
$$

and for all lines of sight $L^{i}, i=1, \ldots N_{L}$ :

$$
\left\{\begin{array}{l}
\frac{d \mathbf{s}^{i}}{d Z^{i}}=\boldsymbol{G}(\psi, \mathcal{B}, \mathcal{C}) \mathbf{s}^{i}, \quad \text { on }\left(Z_{0}^{i}, Z_{1}^{i}\right], \\
\mathbf{s}^{i}\left(Z_{0}^{i}\right)=\mathbf{s}_{0}^{i}
\end{array}\right.
$$


The unknown functions $\mathcal{A}, \mathcal{B}$ and $\mathcal{C}$ are supposed to belong to a set $\mathcal{U}$ of regular functions defined on $[0,1]$.

The formulation in the case of classical Faraday polarimetry measurements consists in droping the Stokes model of Eq. (15) and defining another cost function

$$
J_{F}(\psi, \mathcal{A}, \mathcal{B}, \mathcal{C}):=J_{o F}(\psi, \mathcal{A}, \mathcal{C})+R(\mathcal{A}, \mathcal{B})+R_{C}(\mathcal{C}),
$$

where

$$
J_{o F}(\psi, \mathcal{A}, \mathcal{C}):=J_{\text {mag }}(\psi)+J_{\text {interf }}(\psi, \mathcal{C})+J_{\text {polar } F}(\psi, \mathcal{C})+J_{\text {press }}(\psi, \mathcal{A})
$$

and the polarimatry term uses the classical formulation

$$
J_{\text {polar } F}(\psi, \mathcal{C}):=\sum_{i=1}^{N_{L}} \frac{1}{2 \sigma_{F_{i}}^{2}}\left(\frac{1}{2} W_{3}^{i}(\psi, \mathcal{C})-\delta \alpha_{o b s}^{i}\right)^{2} .
$$

The equilibrium reconstruction problem is then formulated as:

Reconstruction problem 2 (with classical polarimetry) Find $(\psi, \mathcal{A}, \mathcal{B}, \mathcal{C})$ minimizing $J_{F}(\psi, \mathcal{A}, \mathcal{B}, \mathcal{C})$ from Eq. (16) under the constraint of the model equation (14).

Clearly the case where magnetics only are used for the reconstruction is obtained using

$$
J_{M}(\psi, \mathcal{A}, \mathcal{B}):=J_{o M}(\psi)+R(\mathcal{A}, \mathcal{B}),
$$

where

$$
J_{o M}(\psi):=J_{m a g}(\psi)
$$

and the equilibrium reconstruction problem is then formulated as:

Reconstruction problem 3 (magnetics only) Find $(\psi, \mathcal{A}, \mathcal{B})$ minimizing $J_{M}(\psi, \mathcal{A}, \mathcal{B})$ from Eq. (19) under the constraint of the model equation (14).

\subsection{Numerical solution method}

The numerical methods used in this work are the ones presented in $[9$. They have been implemented in a new $\mathrm{C}++$ code called NICE (Newton direct and Inverse Computation for Equilibrium). Without going into technical details we briefly describe the different steps of the algorithm.

Equilibrium equation (14) is discretized using a P1 finite elements method based on a triangular mesh [7, 1, 11. Stokes model for polarimetry is discretized using the Crank-Nicolson scheme for the integration of $(15)$ on each line of sight. Figure 1 displays the discretized domain and lines of sight for JET. The functions to be identified by the reconstruction, $\mathcal{A}, \mathcal{B}$ and $\mathcal{C}$ are decomposed in a basis of typically cubic spline functions $\phi_{i}$ defined on $[0,1]$.

After discretization of the cost function $(10)$ we have to solve a finite dimensional optimization problem involving a non quadratic cost function, under the equality constraint of a non-linear model. This is a achieved thanks to an iterative scheme derived from the sequential quadratric programming method (SQP) [21, 12. The derivation of the scheme is not trivial essentially because of the particular non-linearity of the equilibrium equation due to the free plasma boundary aspect and because of the coupling between the Stokes and equilibrium equations. At convergence of the iterations the optimal $\mathcal{A}, \mathcal{B}$ and $\mathcal{C}$ functions are found and error bars on theses reconstructed functions can be computed from the Hessian matrix of the (reduced) cost function.

In the remaining part of this work a computation solving reconstruction problem 1 is denoted by NICE S, one solving reconstruction problem 2 is denoted by NICE F and finally one solving reconstruction problem 3 is denoted by NICE M 


\section{Application to JET data}

\section{1 data}

JET far-infrared diagnostic is a hybrid interferometer-polarimeter. It provides the line integrated electron density over 8 lines of sight (also called channels). The first one is not considered as input for the reconstructions since it is often outside the plasma domain. For polarimetry we consider only channels 3, 5 and 7 in order to be consistent with the EFIT JET codes. The components of the Stokes vector are related directly to quantities measured by the polarimetric system. The quantites of interest for this study which are stored in the JET polarimetry database are: $\theta$ the angle related to the ratio of the components of the electric field, $\alpha$ the Faraday rotation angle, $\phi$ the Cotton-Mouton angle and $\theta_{0}, \alpha_{0}, \phi_{0}$ their initial prior plasma values, and $E$ the ellipticity from which one can recompute the ellipticity angle defined by $E=\tan (\chi)$. The initial ellipticity angle is $\chi_{0}=0$. From these data, the measured Stokes vector can be computed as:

$$
\begin{cases}s_{1}=\cos (2 \chi) \cos (2 \alpha) & =\cos (2 \theta) \\ s_{2}=\cos (2 \chi) \sin (2 \alpha) & =\sin (2 \theta) \cos (\phi) \\ s_{3}=\sin (2 \chi) & =\sin (2 \theta) \sin (\phi)\end{cases}
$$

The initial Stokes vectors are $(0,1,0)$ for channels $1-2,(0,-1,0)$ for channels $3-4$ and $(1,0,0)$ for channels $5-8$.

The shots selected for this study are $92394,92398,92436$ and 92441 . Their plasma parameters are summarized in Table 1

\begin{tabular}{|c|c|c|c|c|c|}
\hline shot & Ip / Bt & NeL & NBI / ICRH & neutrons & scenario \\
\hline 92394 & $2.2 / 2.8$ & 1.3 & $26.6 / 5.0$ & $2.70 \mathrm{e} 16$ & hybrid \\
92398 & $2.2 / 2.8$ & 1.33 & $27.0 / 5.31$ & $2.72 \mathrm{e} 16$ & hybrid \\
92436 & $3.0 / 2.8$ & 1.74 & $27.3 / 5.6$ & $2.90 \mathrm{e} 16$ & H-mode \\
92441 & $3.5 / 3.0$ & 1.26 & $27.5 / 4.6$ & $1.30 \mathrm{e} 16$ & H-mode \\
\hline
\end{tabular}

Table 1: Plasma parameters of the JET pulses. Ip plasma current in [MA], Bt toroidal magnetic field in [T], NeL line integrated electron density measured on channel 3 of the interferometer in [1e20 $\mathrm{m}^{-2}$, heating power NBI (Neutral beam) and ICRH (ion cyclotron) in [MW], neutron flux in $[\mathrm{n} / \mathrm{s}]$.

The pulses belong to both Hybrid and H-mode scenarios. In particular the pulses 92394, 92398 and 92436 are the record neutron pulses in the 2016 campaigns and likely the basis of the scenario development for the DT campaign. Pulse 92441 has the highest current and magnetic field.

The polarimetry measured maximum parameters are shown on Table 2

\begin{tabular}{|c|c|c|c|}
\hline shot & FAR3 & CMA3 & ELL \\
\hline 92394 & 0.1 & 0.22 & 0.11 \\
92398 & 0.1 & 0.22 & 0.11 \\
92436 & 0.2 & 0.35 & 0.15 \\
92441 & 0.4 & 0.6 & 0.22 \\
\hline
\end{tabular}

Table 2: Polarimetry parameters (max). Measurements of Faraday rotation FAR3 [rad], CottonMouton Phase shiftCMA3 [rad] and ellipticity ELL on channel 3 of the polarimeter 
These shots were chosen to test the equilibrium reconstruction made by the NICE code in high performance pulses, where the low signal approximation of polarimetry constraints is marginal, and then the rigorous solution of the polarimetry propagation equations is needed.

For each of them equilibrium reconstructions NICE M, NICE F and NICE S were performed with a time step of $0.1 \mathrm{~s}$ and one particular instant in time was selected according to the availability of results from EFTM in the JET database (EFIT uses magnetics only and is always available; EFTF uses magnetics, polarimetry and pressure. EFTM uses magnetics, polarimetry, pressure and MSE; the results from EFTF and EFTM are only available for selected time slices).

The Cauchy conditions on $\partial \Omega$ needed as magnetic inputs by NICE are provided by the XLOC JET code. Pressure constraints are the same as those used by EFTM.

\subsection{Time evolution of reconstructed polarimetry quantities}

As a first overwhole aspect of the different simulations we consider the error between measured variation of the Faraday angle and the computed one. For NICE F, this computed variation is given directly by

$$
\delta \alpha_{F}=\frac{1}{2} W_{3} .
$$

whereas for NICE $\mathrm{S}$ it has to be post-processed as

$$
\delta \alpha_{S}=\frac{1}{2} \arctan \left(\frac{s_{2}}{s_{1}}\right)-\alpha_{0}
$$

where $s_{1}$ and $s_{2}$ are the first 2 components of the computed Stokes vector for the channel under consideration. These values have to be compared to the measured value $\delta \alpha_{\text {meas }}$. The mean over the 3 used lines of sight, $3-5-7$, of the errors $\left|\delta \alpha_{F}-\delta \alpha_{\text {meas }}\right|$ and $\left|\delta \alpha_{S}-\delta \alpha_{\text {meas }}\right|$ is plotted for the 4 shots on the left hand side of figures 2, 3, 4, and 5. It appears clearly that globally the errors for NICE $\mathrm{S}$ are lower than the errors for NICE F. This results is particularly striking since $\delta \alpha_{\text {meas }}$ is the quantity directly involved in the polarimetry least square term of $J_{F}$, the cost function for NICE F, whereas it is not the case for NICE S where the measured Stokes vector is used instead in $J_{S}$.

The errors on ellipticity are shown on the right hand side of the same figures. For NICE F the ellipticity is approximated as

$$
E_{F}=-\frac{1}{2} W_{1} \quad \text { or } \quad E_{F}=-\frac{1}{2} W_{2}
$$

depending on the initial Stokes vector, whereas for NICE S it is computed as

$$
E_{S}=\tan \left(\frac{1}{2} \arcsin \left(s_{3}\right)\right)
$$

The same improvement is observed for NICE S compared to NICE F.

Figures 6 and 7 show the chord by chord time evolution of Faraday angle and ellipticity as well as the relative errors for NICE F and S for shot 92394. In particular it can be observed that the improvement on ellipticity in NICE S mainly results from chords 5 and 7 on which NICE F does not perform well, whereas the relative errors for these chords in NICE $\mathrm{S}$ fall to the level of what is obtained on chord 3 .

\subsection{Error bars on reconstructed profiles}

Figures 8, 9, 10, and 11 show the reconstructed $p^{\prime}$ and $f f^{\prime}$ profiles as well as their computed error bars at one instant in time for each of the 4 shots. In NICE M functions $\mathcal{A}$ and $\mathcal{B}$ (hence 
$p^{\prime}$ and $f f^{\prime}$ ) are forced to be 0 on the plasma boundary where $\psi_{\mathrm{N}}=1$. On the constrary in NICE $\mathrm{F}$ and $\mathrm{S}$ this boundary value is left free. From these figures the awaited fact that the use of interferometry, polarimetry and pressure constraints in addition to magnetics decreases the error bars, appears clearly. It also appears that the use of Stokes vector measurements instead of classical Faraday measurements decreases the error bars in the central part of the plasma $\left(\psi_{\mathrm{N}} \leq 0.5\right)$. Even though the differences in the reconstructed profiles in NICE F or NICE S are not extremely important, the computed error bars are always lower for NICE $\mathrm{S}$ than for NICE $\mathrm{F}$ and NICE M meaning that the equilibrium reconstruction is better constrained.

\subsection{Fit to interferometry and polarimetry measurements}

Since the EFIT codes are well-established and have been calibrated at JET for a long time, we use them as a comparison to check our results. EFIT is always available while EFTM is only available for selected time slices. EFTM uses MSE internal measurements and should thus provide the best approximation to the plasma current density profile.

Although this is difficult to quantify, in general the reconstructed profiles from NICE M and EFIT are quite similar as well as those from NICE S and EFTM. Figures $13,14,15$ and 16 show the reconstructed $p^{\prime}, f f^{\prime}$ and $p, f$ profiles for NICE M, F and $\mathrm{S}$ as well as for EFIT and EFTF/EFTM when available. Table 3 shows the mean relative error on Faraday rotation angle. Going from NICE $\mathrm{F}$ to $\mathrm{S}$ this error clearly decreases, dropping to levels close to EFTM. This is very interesting since NICE $\mathrm{S}$ does not make use of all the MSE points used to constrain the equilibrium in EFTM.

\begin{tabular}{|c|c|c|c|}
\hline shot [time] & NICE F & NICE S & EFTM \\
\hline $92394[47]$ & 0.202 & 0.101 & 0.072 \\
$92398[47]$ & 0.169 & 0.088 & 0.088 \\
$92436[48]$ & 0.151 & 0.092 & 0.081 \\
$92441[50]$ & 0.129 & 0.096 & $\times$ \\
\hline
\end{tabular}

Table 3: Mean relative errors on Faraday rotation angle (mean over used lines of sight: 3-5-7).

The identified plasma boundary does not vary much between NICE M, F and S and is very close from the plasma boundary given by EFIT. However we noticed that often the plasma boundaries from EFTF and EFTM can differ quite significantly between them and from the one given by EFIT. This behavior from EFTF and EFTM is questionable since the plasma boundary should only depend on magnetic measurements. An example of these plasma boundaries is given on Fig. 12 for shot 92398. The boundaries from EFTF and EFTM particularly differ from the others on the high low field side of the plasma.

Table 4 shows that the mean relative error on interferometry does not change significantly between NICE F and S, and stays below $4 \%$ for the 4 time slices and shots.

\begin{tabular}{|c|c|c|}
\hline shot [time] & NICE F & NICE S \\
\hline $92394[47]$ & 0.022 & 0.020 \\
$92398[47]$ & 0.019 & 0.021 \\
$92436[48]$ & 0.015 & 0.040 \\
$92441[50]$ & 0.022 & 0.023 \\
\hline
\end{tabular}

Table 4: Mean relative errors on interferometry (mean over used lines of sight: 2 to 8).

Table 5 shows the mean relative error on ellipticity. These are quite high for NICE F in which 
the computed ellipticity for the horizontal chords 5 and 7 in particular is much smaller than the data (see fig 7). The errors are largely decreased in NICE S but however remain globally higher than the errors on Faraday angles.

\begin{tabular}{|c|c|c|}
\hline shot [time] & NICE F & NICE S \\
\hline $92394[47]$ & 0.663 & 0.276 \\
$92398[47]$ & 0.644 & 0.221 \\
$92436[48]$ & 0.645 & 0.314 \\
$92441[50]$ & 0.683 & 0.474 \\
\hline
\end{tabular}

Table 5: Mean relative errors on ellipticity (mean over used lines of sight: $3-5-7$ ). The errors for NICE F are particularly high because of the ellipticity on chords 5 and 7

\section{Conclusion}

In this work we have presented the first application to real JET data of the new equilibrium code NICE which enables the consistent resolution of the inverse equilibrium reconstruction problem in the framework of non-linear free-boundary equilibrium coupled to the Stokes model equation for polarimetry.

The conducted numerical experiments have enabled first of all to validate NICE by comparing it to the well-estabished EFIT code on 4 selected high performance shots. Secondly the results have indicated that the fit to polarimetry measurements clearly benefits from the use of Stokes vector measurements compared to the classical case of Faraday measurements. The reconstructed profiles from NICE S have appeared to be better constrained than those from NICE M and F with smaller error bars. Morevover they have appeared to be closer from the profiles reconstructed by EFTM the EFIT JET code using internal MSE constraints. This highlights the usefulness of polarimetry measurements with their Stokes model representation in equilibrium reconstruction.

\section{Acknowledgment}

We would like to thank Chiara Piron for her help in providing the XLOC inputs necessary for this study, and Alexandru Boboc for his explanations on JET polarimetry database.

\section{References}

[1] R. Albanese, J. Blum, and O. Barbieri. On the solution of the magnetic flux equation in an infinite domain. In EPS. 8th Europhysics Conference on Computing in Plasma Physics (1986), pages 41-44, 1986.

[2] J. Blum. Numerical Simulation and Optimal Control in Plasma Physics with Applications to Tokamaks. Series in Modern Applied Mathematics. Wiley Gauthier-Villars, Paris, 1989.

[3] J. Blum, C. Boulbe, and B. Faugeras. Real-time plasma equilibrium reconstruction in a tokamak. In Journal of Physics: Conference Series. Proceedings of the 6th International Conference on Inverse Problems in Engineering: Theory and Practice, volume 135, page 012019. IOP Publishing, 2008. 
[4] J. Blum, C. Boulbe, and B. Faugeras. Reconstruction of the equilibrium of the plasma in a tokamak and identification of the current density profile in real time. Journal of Computational Physics, 231(3):960 - 980, 2012.

[5] J Blum and H. Buvat. An inverse problem in plasma physics: the identification of the current density profile in a Tokamak. In Biegler, Coleman, Conn, and Santosa, editors, IMA Volumes in Mathematics and its Applications, Volume 92, "Large Scale Optimization with applications", Part 1: Optimization in inverse problems an design, pages 17-36. Springer, New York, 1997.

[6] J. Blum, E. Lazzaro, J. O'Rourke, B. Keegan, and Y. Stefan. Problems and methods of selfconsistent reconstruction of tokamak equilibrium profiles from magnetic and polarimetric measurements. Nuclear Fusion, 30(8):1475, 1990.

[7] J. Blum, J. Le Foll, and B. Thooris. The self-consistent equilibrium and diffusion code SCED. Comput. Phys. Comm., 24:235 - 254, 1981.

[8] B. Faugeras, J. Blum, C. Boulbe, P. Moreau, and E. Nardon. 2D interpolation and extrapolation of discrete magnetic measurements with toroidal harmonics for equilibrium reconstruction in a Tokamak. Plasma Phys. Control Fusion, 56:114010, 2014.

[9] B. Faugeras, J. Blum, H. Heumann, and C. Boulbe. Optimal control of a coupled partial and ordinary differential equations system for the assimilation of polarimetry stokes vector measurements in tokamak free-boundary equilibrium reconstruction with application to ITER. Comput. Phys. Comm., 217(Supplement C):43 - 57, 2017.

[10] H. Grad and H. Rubin. Hydromagnetic equilibria and force-free fields. Proceedings of the 2nd UN Conf. on the Peaceful Uses of Atomic Energy, 31:190, 1958.

[11] H. Heumann, J. Blum, C. Boulbe, B. Faugeras, G. Selig, J.-M. Ané, S. Brémond, V. Grangirard, P. Hertout, and E. Nardon. Quasi-static free-boundary equilibrium of toroidal plasma with CEDRES ++: computational methods and applications. J. Plasma Physics, 2015.

[12] M. Hinze, R. Pinnau, M. Ulbrich, and S. Ulbrich. Optimization with PDE constraints, volume 23 of Mathematical Modelling: Theory and Applications. Springer, New York, 2009.

[13] F. Hofmann and G. Tonetti. Tokamak equilibrium reconstruction using Faraday rotation measurements. Nuclear Fusion, 28(10):1871, 1988.

[14] L.L. Lao. Separation of $\beta_{p}$ and $l_{i}$ in tokamaks of non-circular cross-section. Nuclear Fusion, 25(11):1421, 1985.

[15] L.L. Lao, J.R. Ferron, R.J. Geoebner, W. Howl, H.E. St. John, E.J. Strait, and T.S. Taylor. Equilibrium analysis of current profiles in Tokamaks. Nuclear Fusion, 30(6):1035, 1990.

[16] R. Lüst and A. Schlüter. Axialsymmetrische magnetohydrodynamische Gleichgewichtskonfigurationen. Z. Naturforsch. A, 12:850-854, 1957.

[17] J.L. Luxon and B.B. Brown. Magnetic analysis of non-circular cross-section tokamaks. Nuclear Fusion, 22(6):813-821, 1982.

[18] P. J. Mc Carthy. Analytical solutions to the Grad-Shafranov equation for tokamak equilibrium with dissimilar source functions. Physics of Plasmas, 6(9):3554-3560, 1999. 
[19] V.V Mirnov, W.X. Ding, D.L. Brower, M.A. Van Zeeland, and T.N. Carlstrom. Finite electron temperature effects on interferometric and polarimetric measurements in fusion plasmas. Phys. Plasmas, 14:102105, 2007.

[20] J.-M. Moret, B.P. Duval, H.B. Le, S. Coda, F. Felici, and H. Reimerdes. Tokamak equilibrium reconstruction code LIUQE and its real time implementation. Fusion Eng. Design, 91(0):1-15, 2015.

[21] J. Nocedal and S. J. Wright. Numerical optimization. Springer Series in Operations Research and Financial Engineering. Springer, New York, second edition, 2006.

[22] D.P. O'Brien, J.J Ellis, and J. Lingertat. Local expansion method for fast plasma boundary identification in JET. Nuclear Fusion, 33(3):467-474, 1993.

[23] F.P. Orsitto, A. Boboc, P. Gaudio, M. Gelfusa, E. Giovannozzi, C. Mazzotta, A. Murari, and JET EFDA Contributors. Mutual interaction of faraday rotation and cotton-mouton phase shift in JET polarimetric measurements. Rev. Sci. Instrum., 81:10D533, 2010.

[24] F.P. Orsitto, A. Boboc, P. Gaudio, M. Gelfusa, E. Giovannozzi, C. Mazzotta, A. Murari, and JET EFDA Contributors. Analysis of faraday rotation in JET polarimetry measurements. Plasma Phys. Control Fusion, 53:035001, 2011.

[25] F.P. Orsitto, A. Boboc, C. Mazzotta, E. Giovannozzi, L. Zabeo, and JET EFDA Contributors. Modelling of polarimetry measurements at JET. Plasma Phys. Control Fusion, 50:115009, 2008 .

[26] V.D. Pustovitov. Magnetic diagnostics: General principles and the problem of reconstruction of plasma current and pressure profiles in toroidal systems. Nuclear Fusion, 41(6):721, 2001.

[27] Sergio E Segre. A review of plasma polarimetry - theory and methods. Plasma Phys. Control Fusion, 41(2):R57, 1999.

[28] V D Shafranov. Determination of the parameters $\beta_{p}$ and $l_{i}$ in a tokamak for arbitrary shape of plasma pinch cross-section. Plasma Physics, 13(9):757, 1971.

[29] V.D. Shafranov. On magnetohydrodynamical equilibrium configurations. Soviet Journal of Experimental and Theoretical Physics, 6:545, 1958.

[30] D.W. Swain and G.H. Neilson. An efficient technique for magnetic analysis for non-circular, high-beta tokamak equilibria. Nuclear Fusion, 22(8):1015-1030, 1982.

[31] L.E. Zakharov and V.D. Shafranov. Equilibrium of a toroidal plasma with noncircular cross-section. Sov. Phys. Tech. Phys., 18(2):151-156, 1973.

[32] W. Zwingmann. Equilibrium analysis of steady state tokamak discharges. Nuclear Fusion, 43:842-850, 2003. 


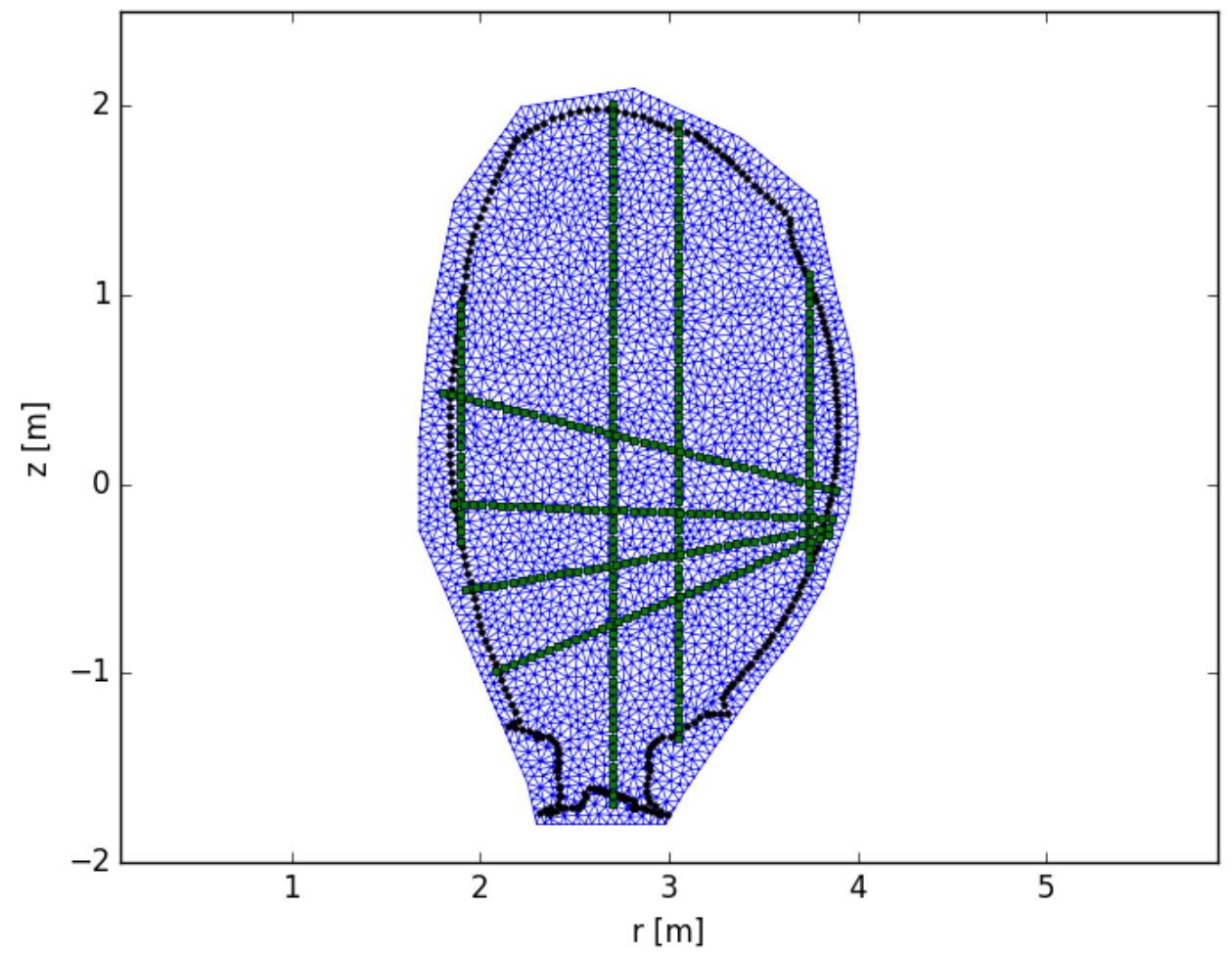

Figure 1: Example of finite element mesh used at JET. In black the limiter contour. In green the 8 discretized interferometry and polarimetry lines of sight (numbered 1 to 4 for the vertical ones from left to right, and 5 to 8 from bottom to top for the others). 

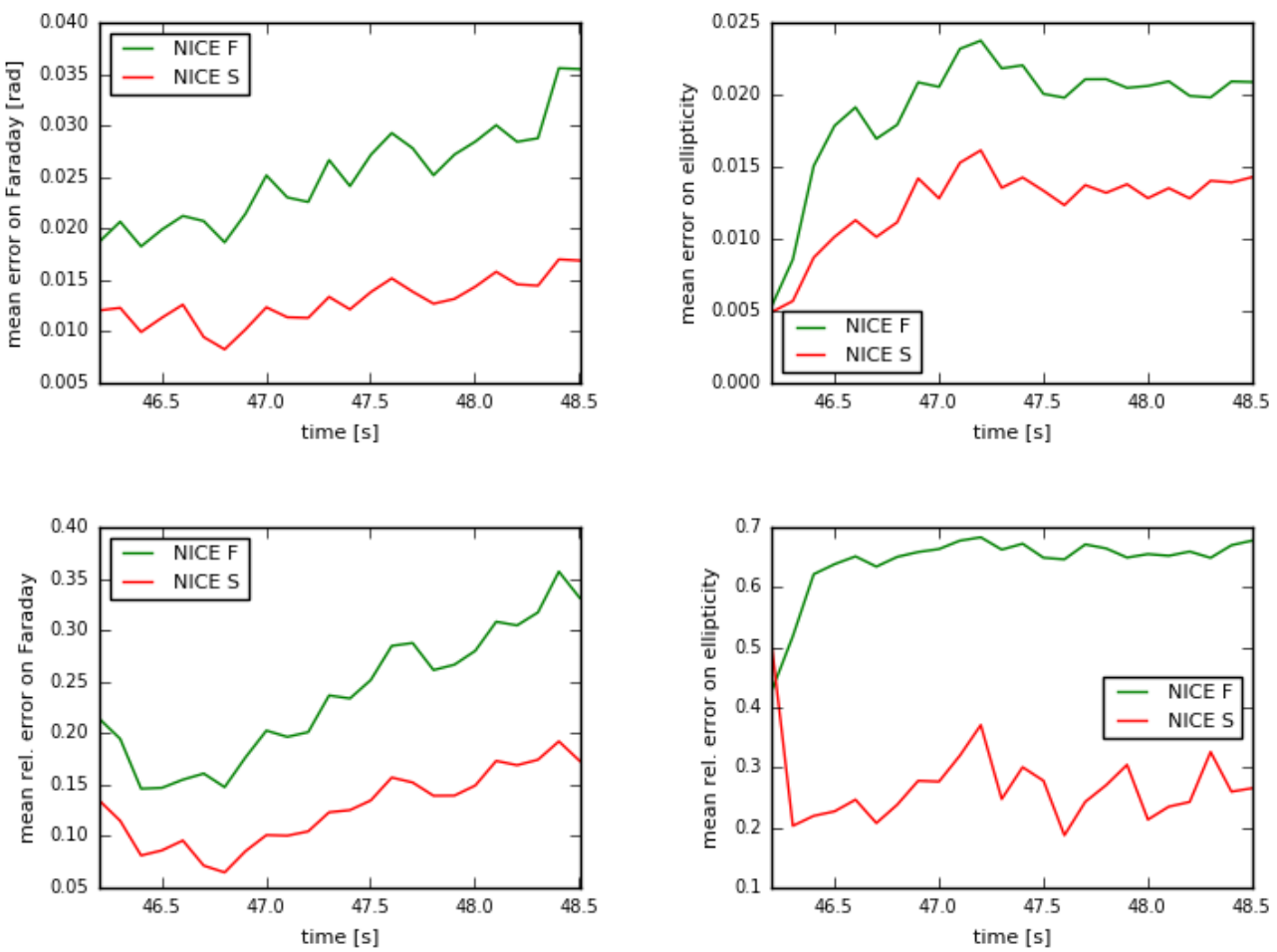

Figure 2: Shot 92394. Row 1: mean over the 3 used polarimetry lines of sight $(3,5,7)$ of the error between computed and measured Faraday angle (left) for NICE F (green) and NICE S (red) and for the ellipticity (right). Row 2: same for the relative error. 

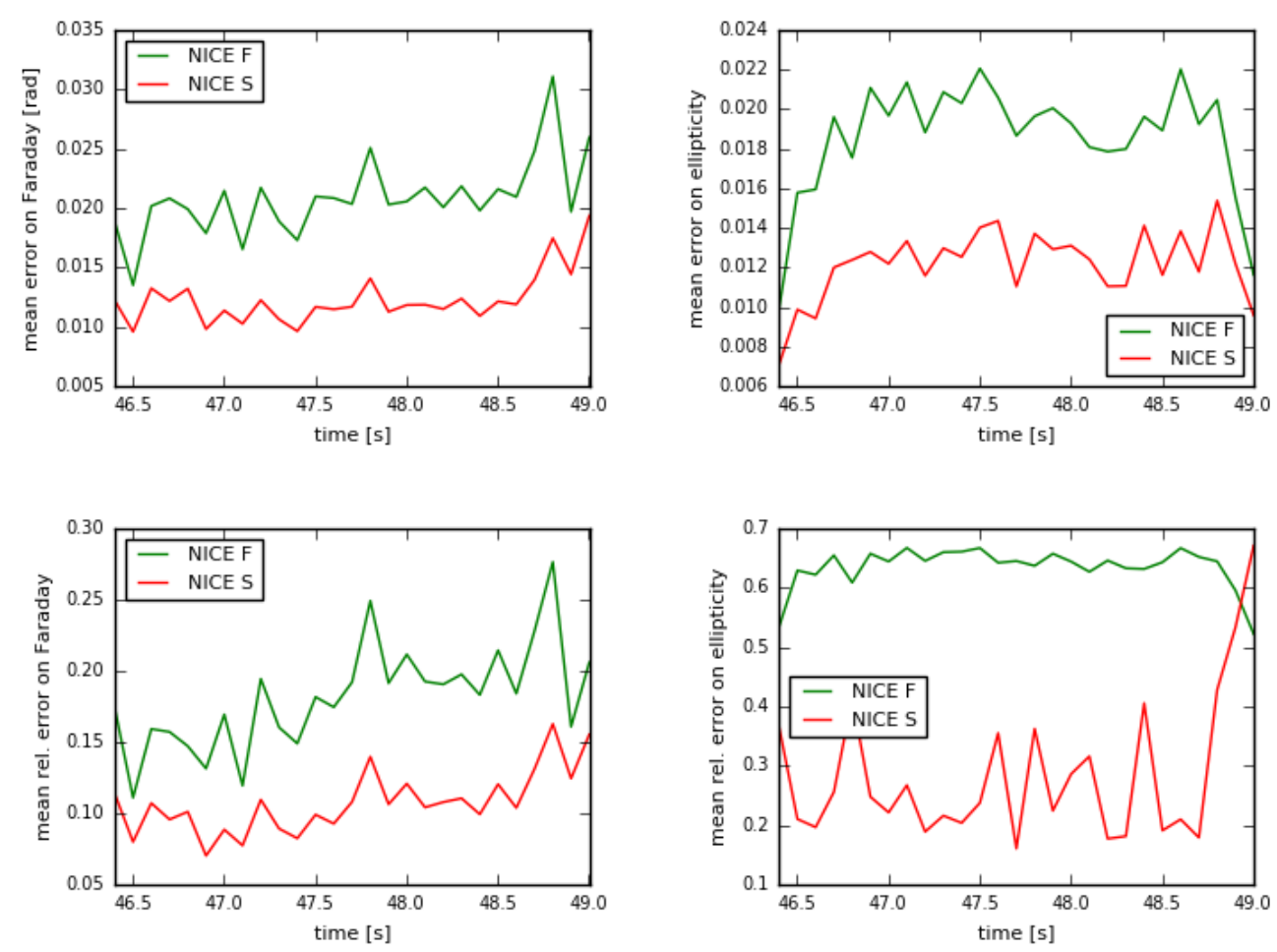

Figure 3: Shot 92398. Row 1: mean over the 3 used polarimetry lines of sight $(3,5,7)$ of the error between computed and measured Faraday angle (left) for NICE F (green) and NICE S (red) and for the ellipticity (right). Row 2: same for the relative error. 

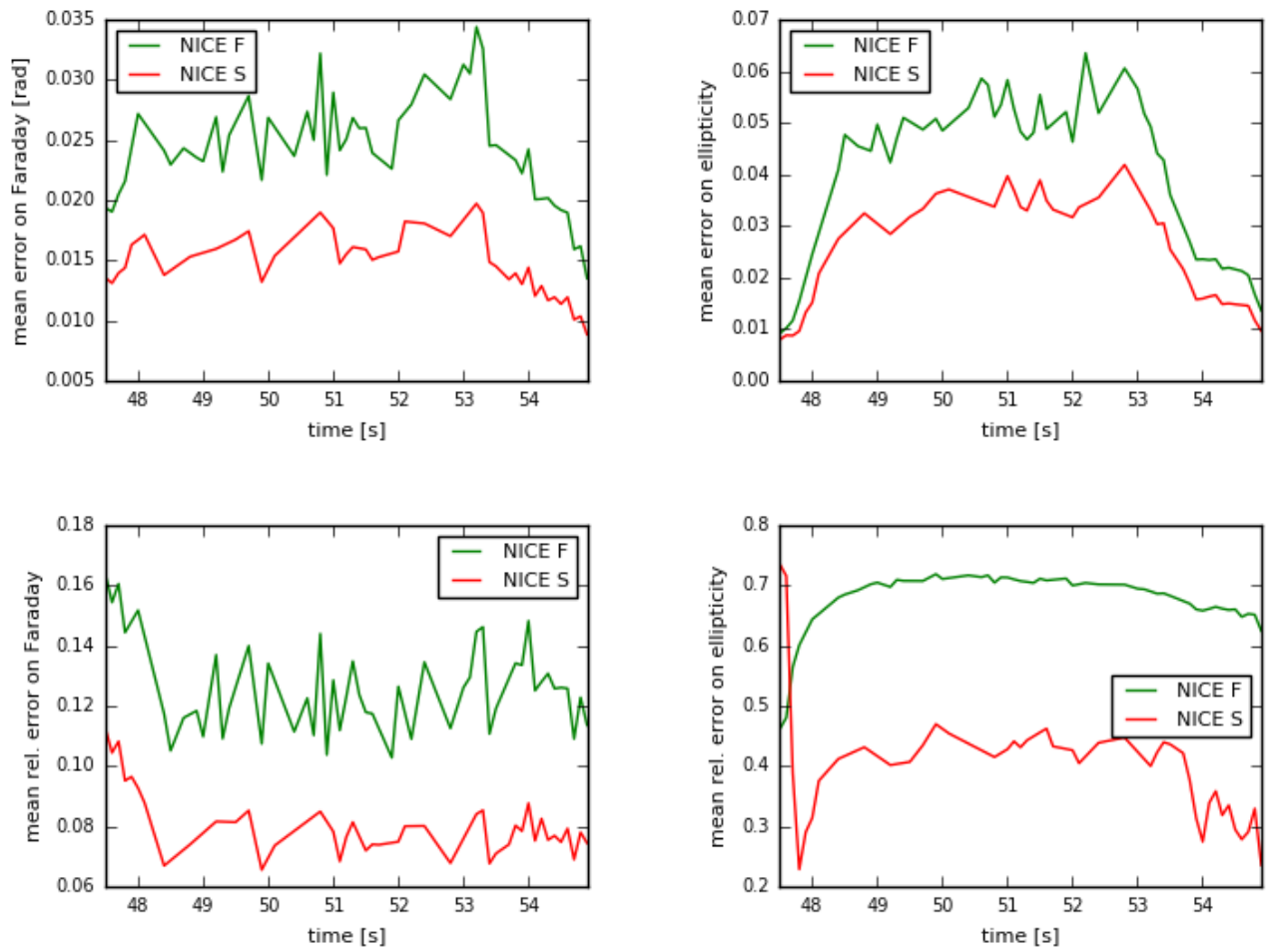

Figure 4: Shot 92436. Row 1: mean over the 3 used polarimetry lines of sight $(3,5,7)$ of the error between computed and measured Faraday angle (left) for NICE F (green) and NICE S (red) and for the ellipticity (right). Row 2: same for the relative error. 

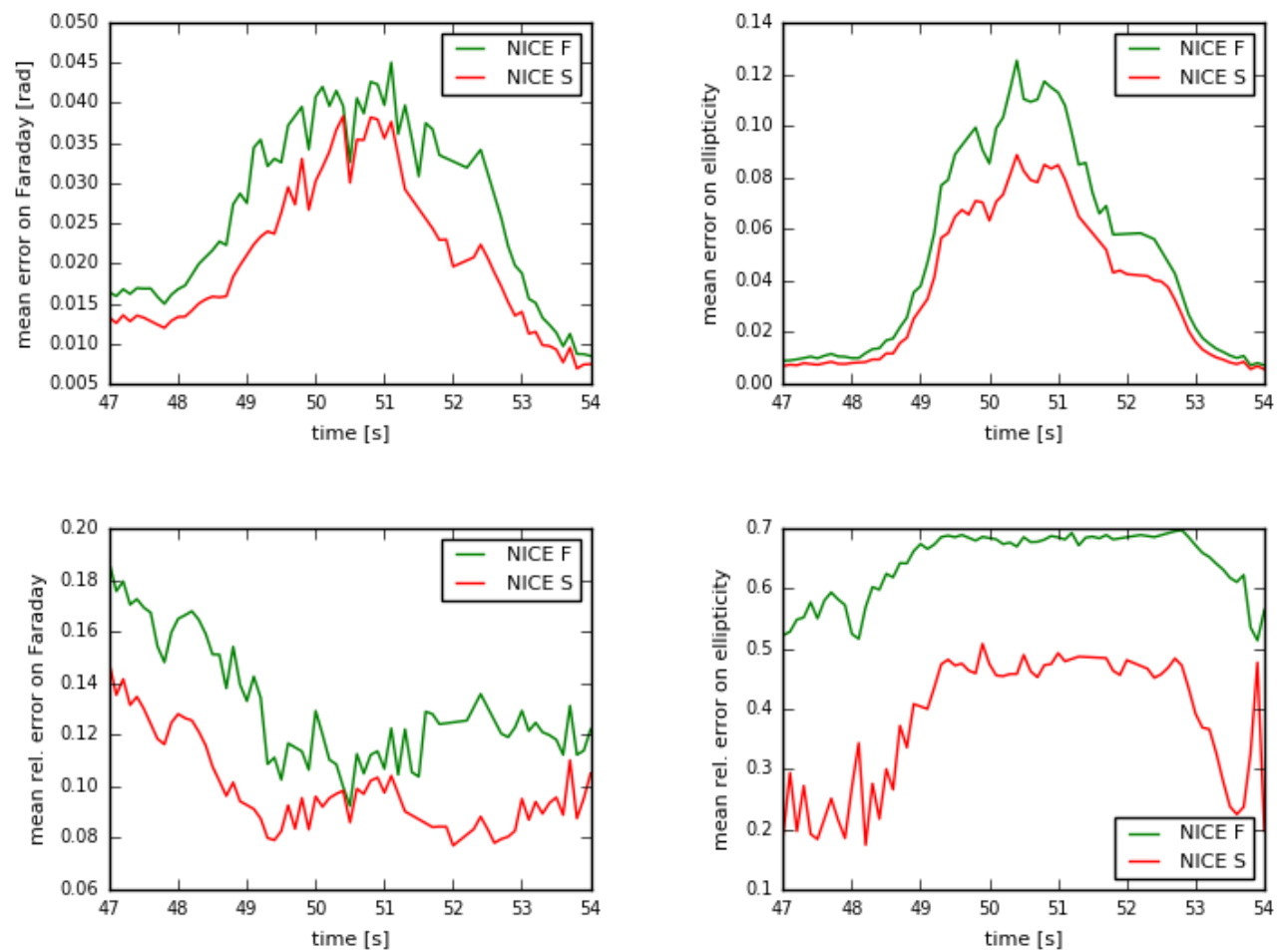

Figure 5: Shot 92441. Row 1: mean over the 3 used polarimetry lines of sight $(3,5,7)$ of the error between computed and measured Faraday angle (left) for NICE F (green) and NICE S (red) and for the ellipticity (right). Row 2: same for the relative error. 

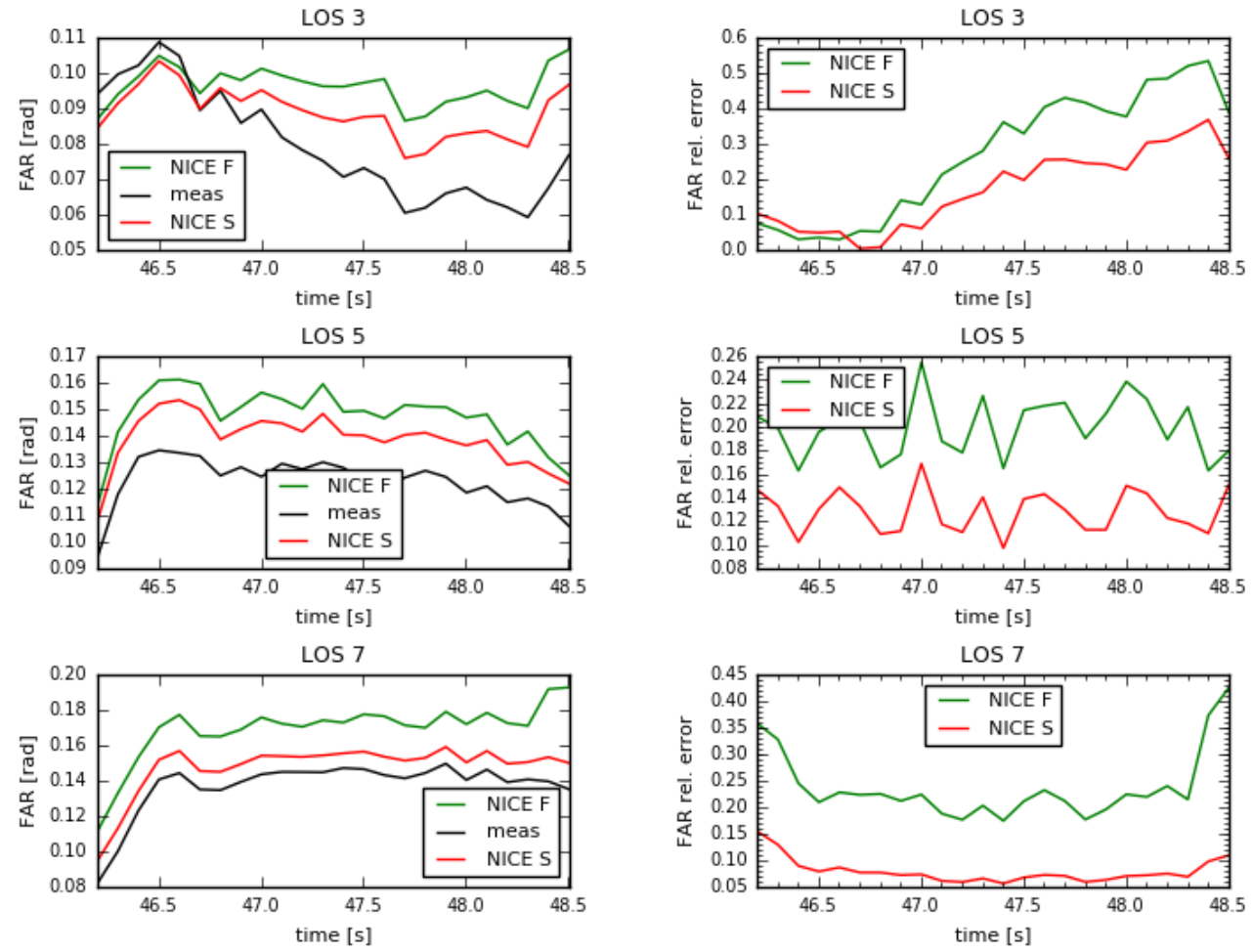

Figure 6: Shot 92394. Faraday angle. Measured (black), NICE F (green), NICE S (red). Relative error for NICE F and S on second column. Line of sight 3-5-7 by row. 

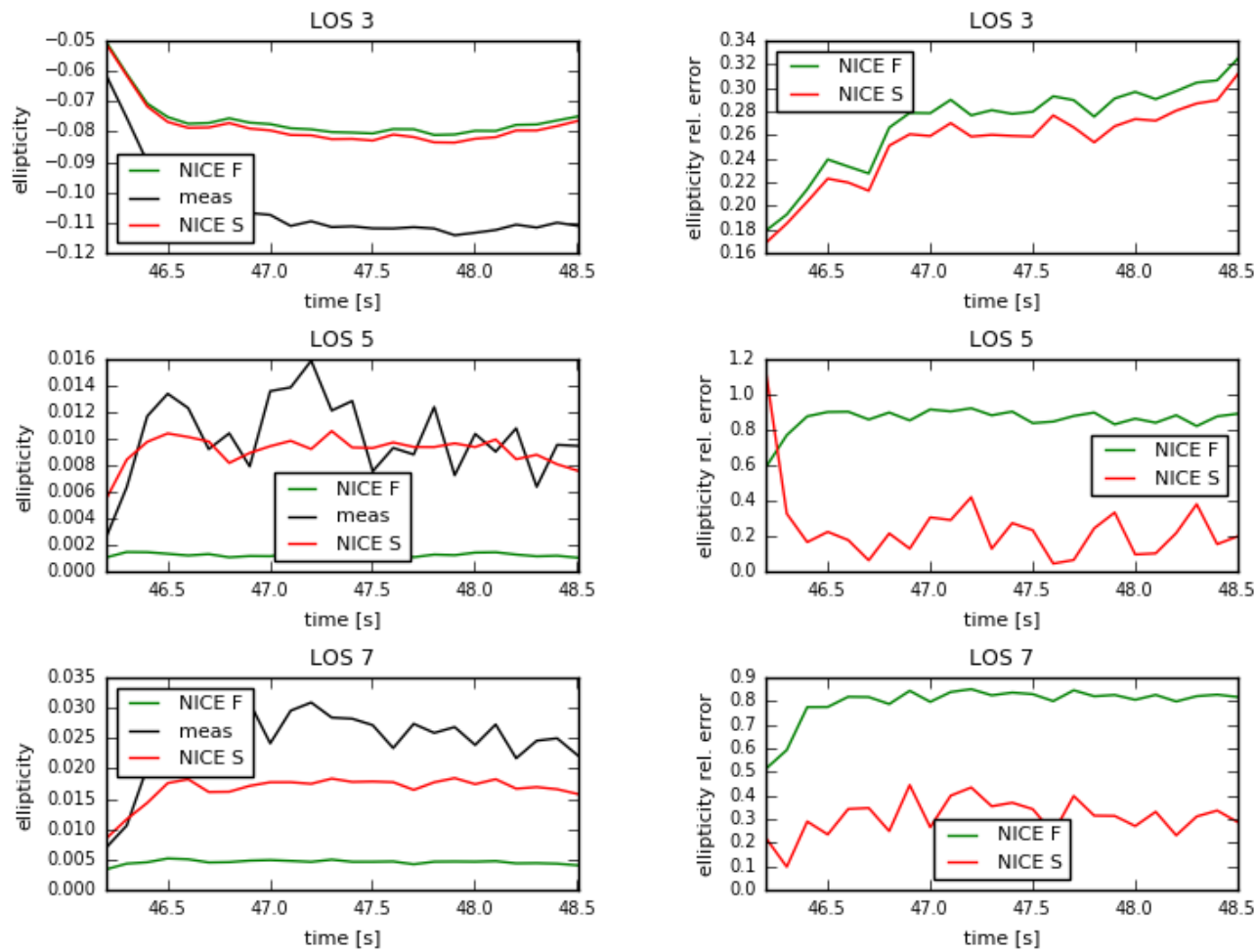

Figure 7: Shot 92394. Ellipticity. Measured (black), NICE F (green), NICE S (red). Relative error for NICE $\mathrm{F}$ and $\mathrm{S}$ on second column. Line of sight 3-5-7 by row. 

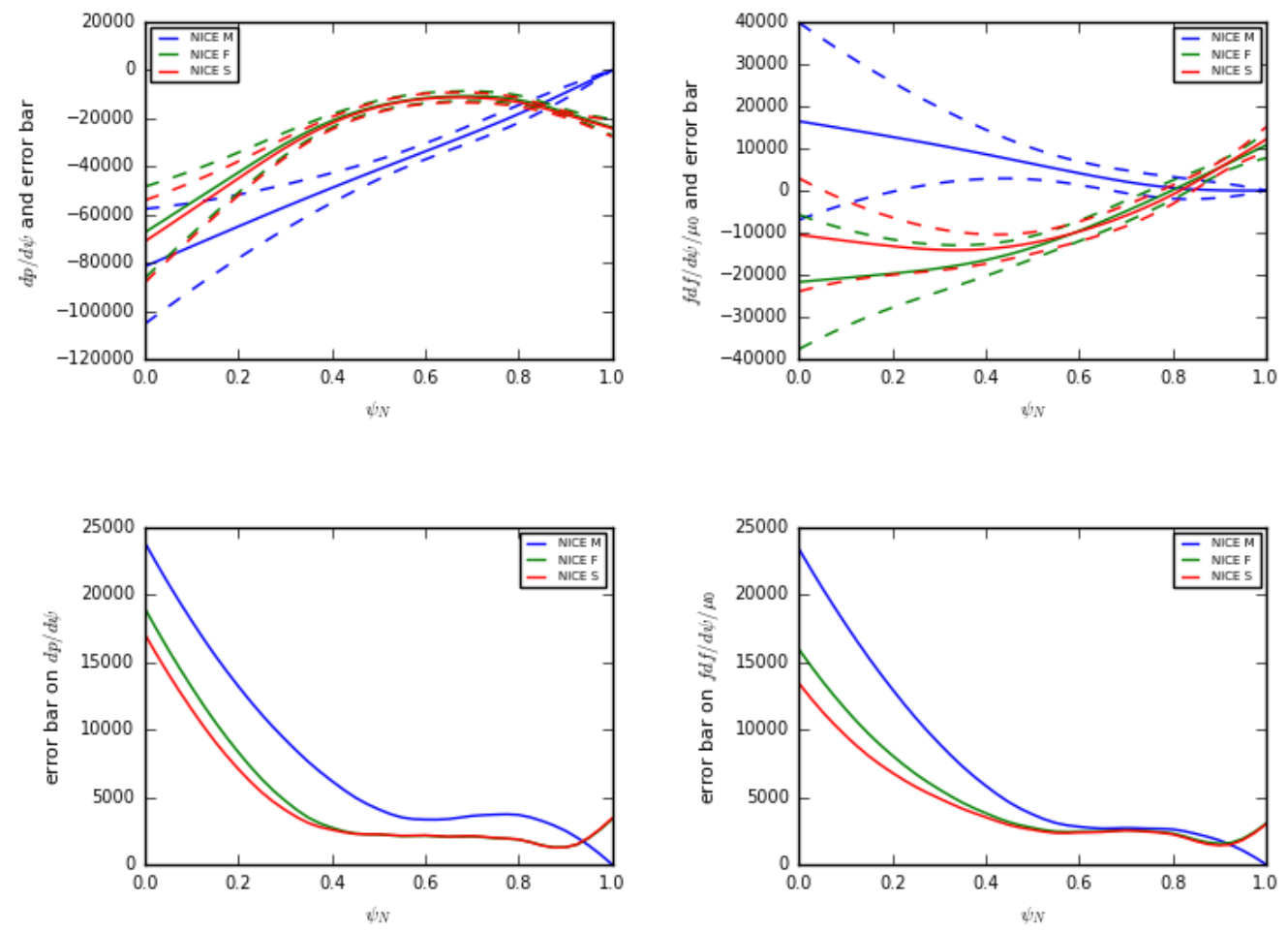

Figure 8: Shot 92394, time 47s. Reconstructed $p^{\prime}$ (top left), $f f^{\prime}$ (top right) \pm computed error bars (dashed) profiles. Blue (NICE M), green (NICE F), red (NICE S). The error bar profiles alone are shown on the bottom row. 

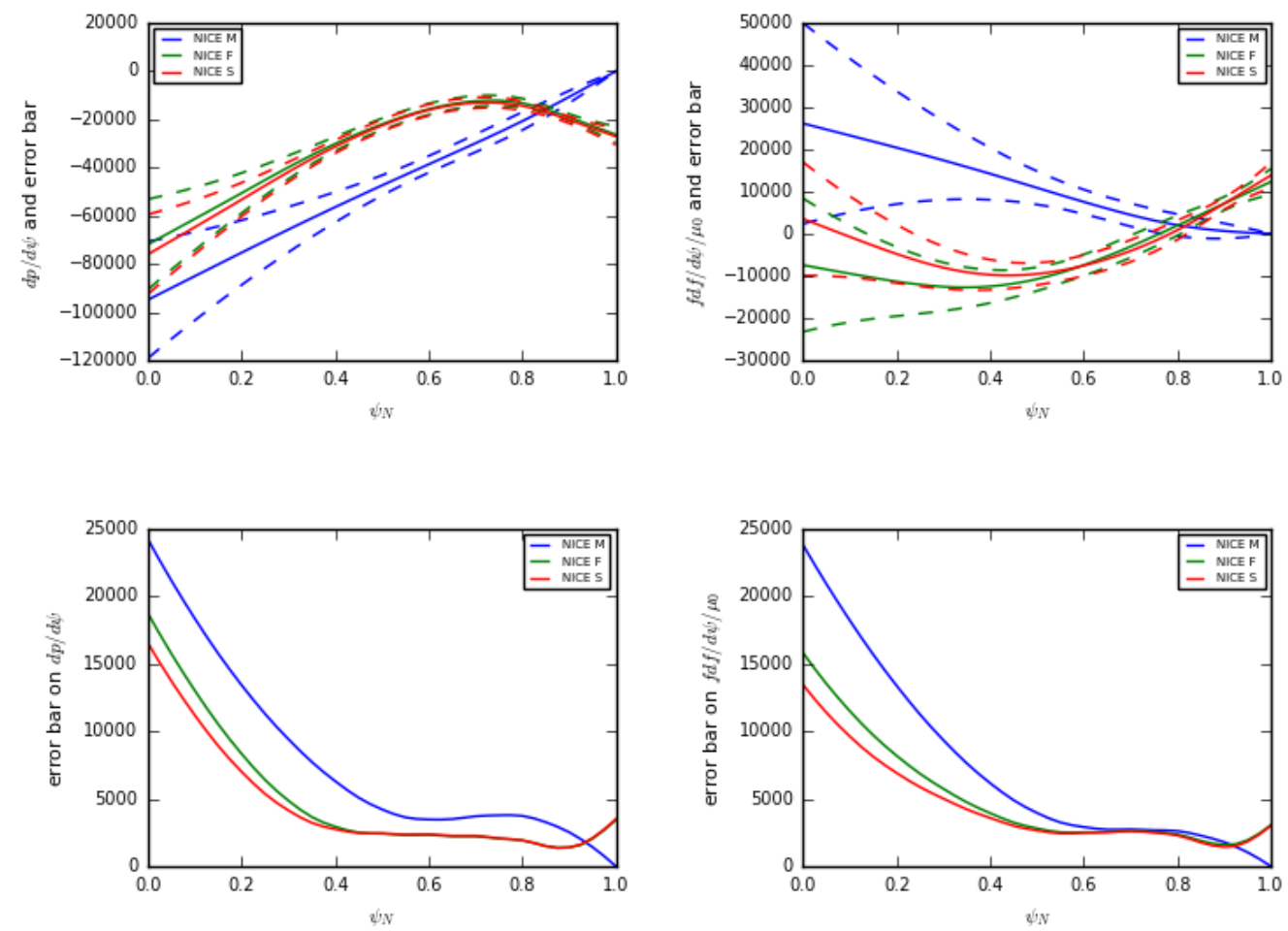

Figure 9: Shot 92398, time 47s. Reconstructed $p^{\prime}$ (top left), $f f^{\prime}$ (top right) \pm computed error bars (dashed) profiles. Blue (NICE M), green (NICE F), red (NICE S). The error bar profiles alone are shown on the bottom row. 

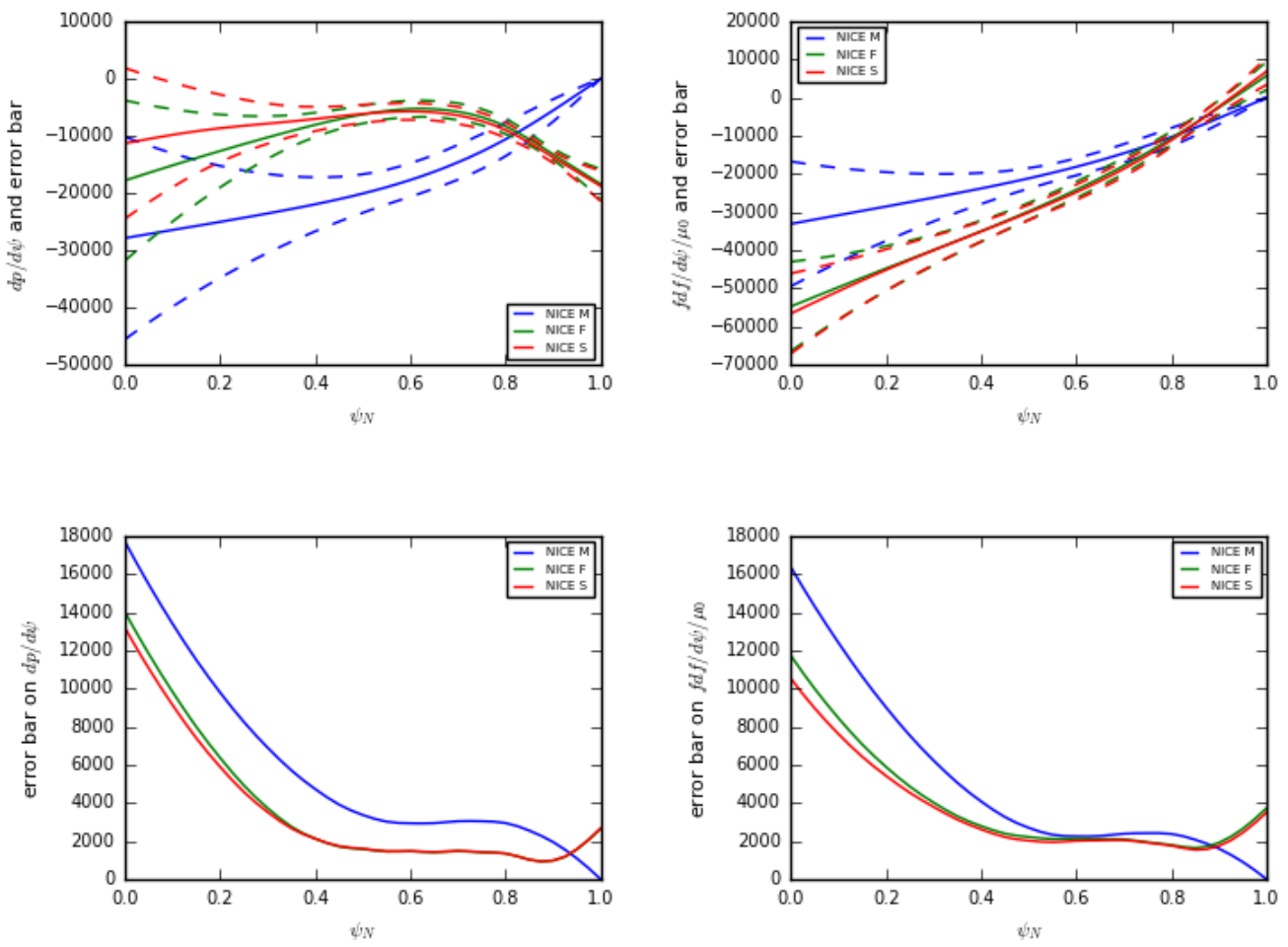

Figure 10: Shot 92436, time 48s. Reconstructed $p^{\prime}$ (top left), $f f^{\prime}$ (top right) \pm computed error bars (dashed) profiles. Blue (NICE M), green (NICE F), red (NICE S). The error bar profiles alone are shown on the bottom row. 

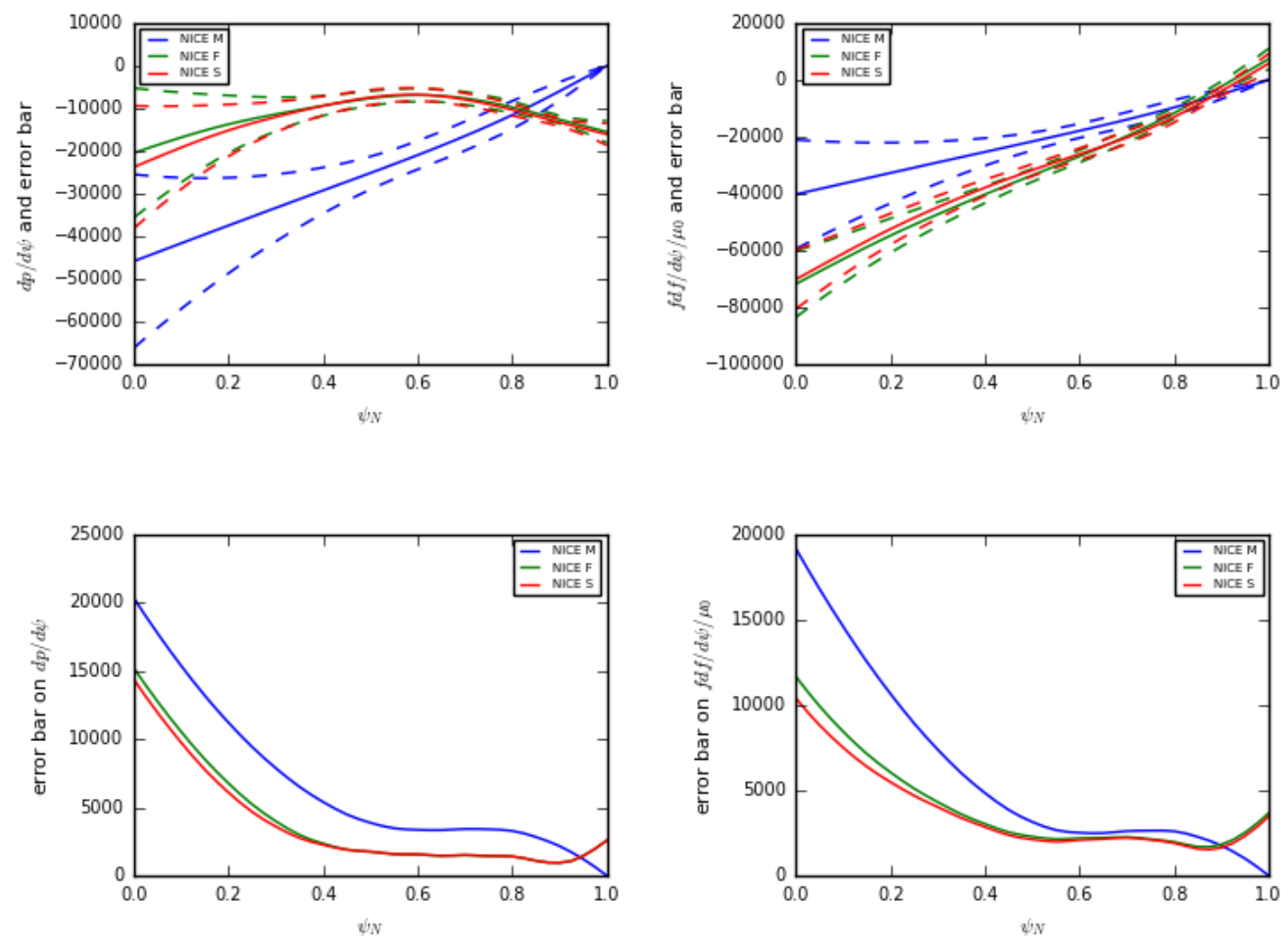

Figure 11: Shot 92441, time 50s. Reconstructed $p^{\prime}$ (top left), $f f^{\prime}$ (top right) \pm computed error bars (dashed) profiles. Blue (NICE M), green (NICE F), red (NICE S). The error bar profiles alone are shown on the bottom row. 


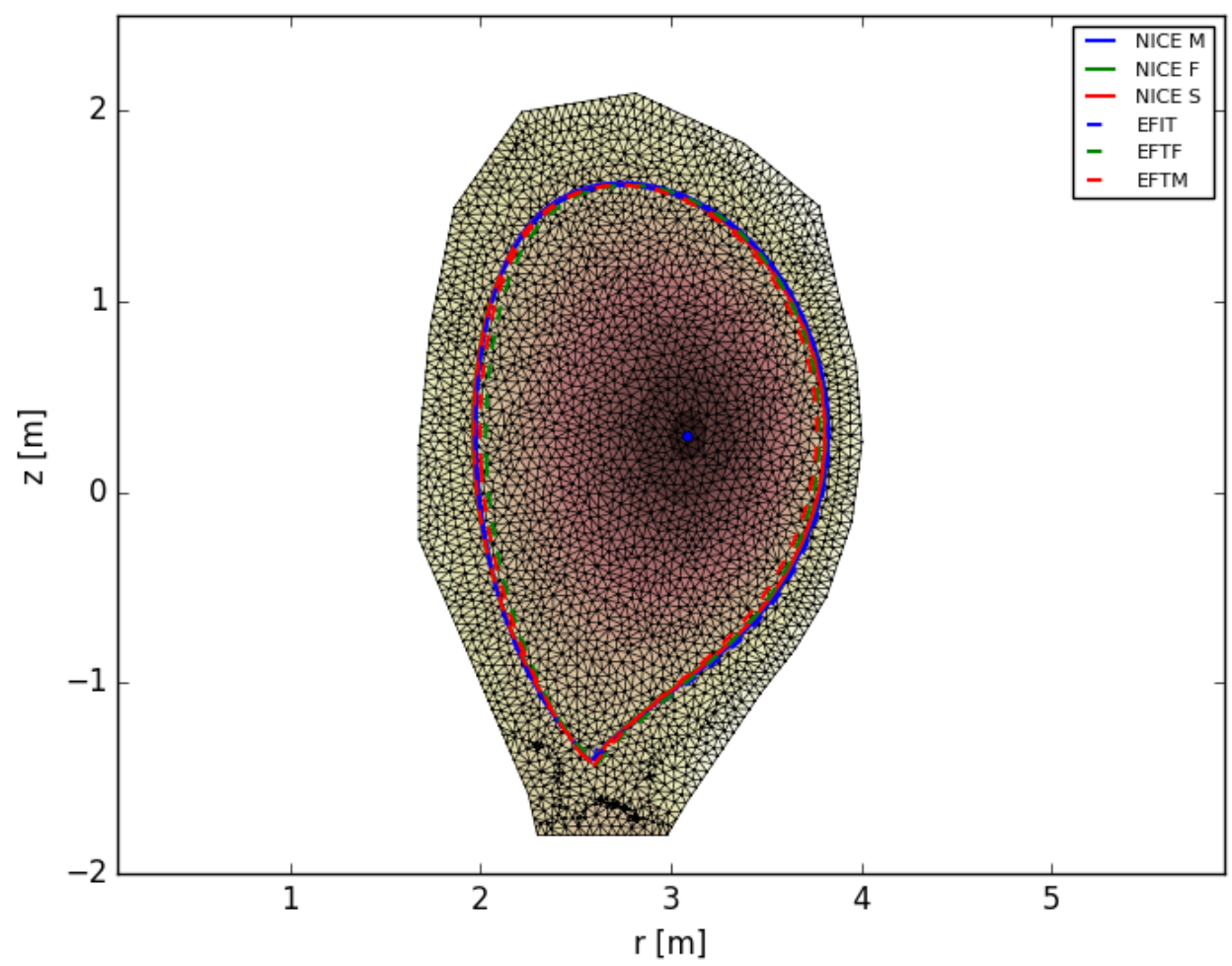

Figure 12: Shot 92398, time 47s. The 2d poloidal flux map is shown for case M (magnetics only). Also shown the plasma boundaries computed by NICE M (continuous blue), NICE F (continuous green) and NICE S (continuous red), EFIT (dashed blue), EFTF (dashed green) and EFTM (dashed red). 

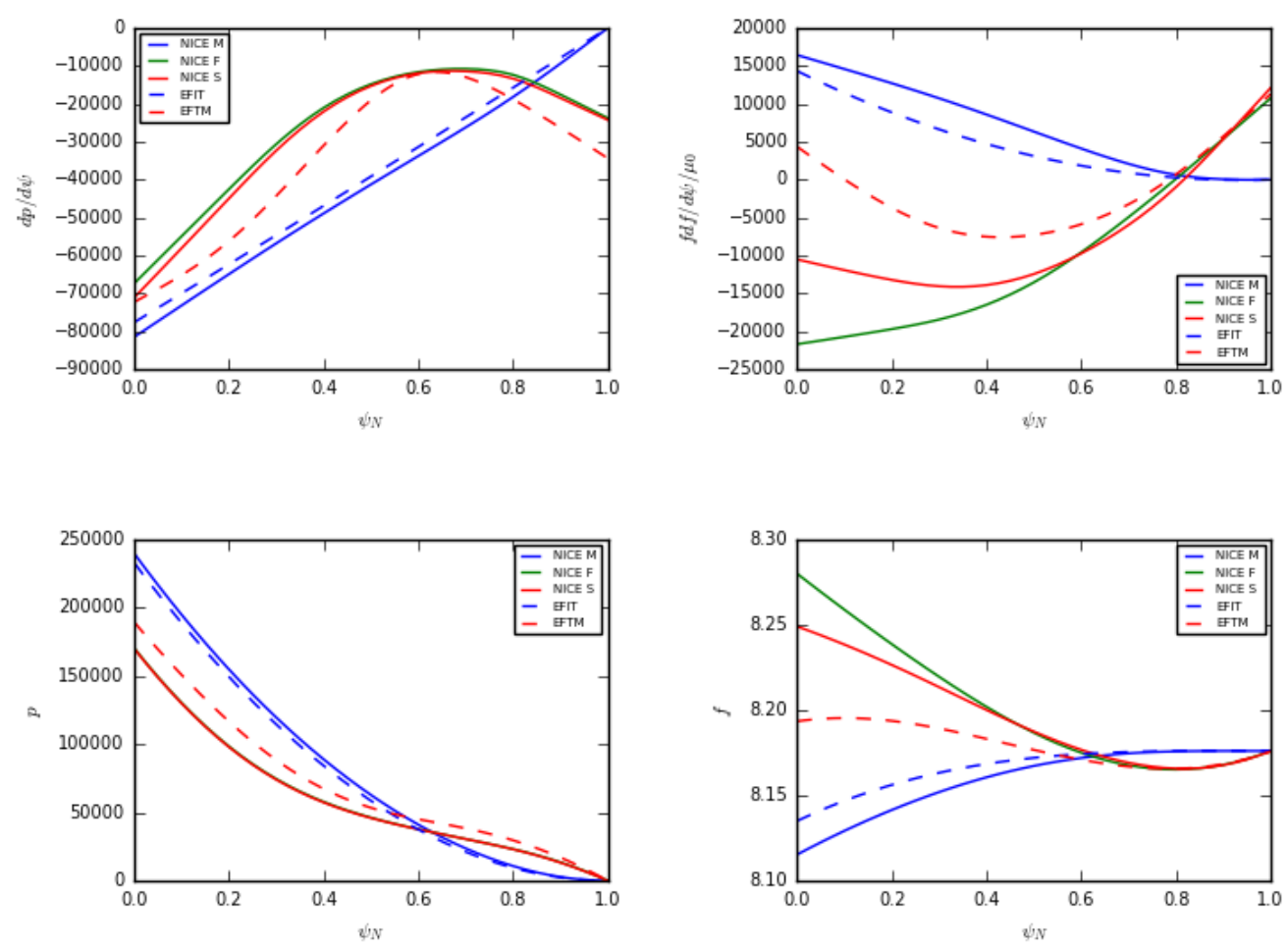

Figure 13: Shot 92394, time 47s. $p^{\prime}, f f^{\prime}$ (first row) and $p, f$ (second row) reconstructed profiles as function of the normalized flux $\psi_{N}$. NICE M (continuous blue), NICE F (continuous green) and NICE S (continuous red), EFIT (dashed blue) and EFTM (dashed red). 

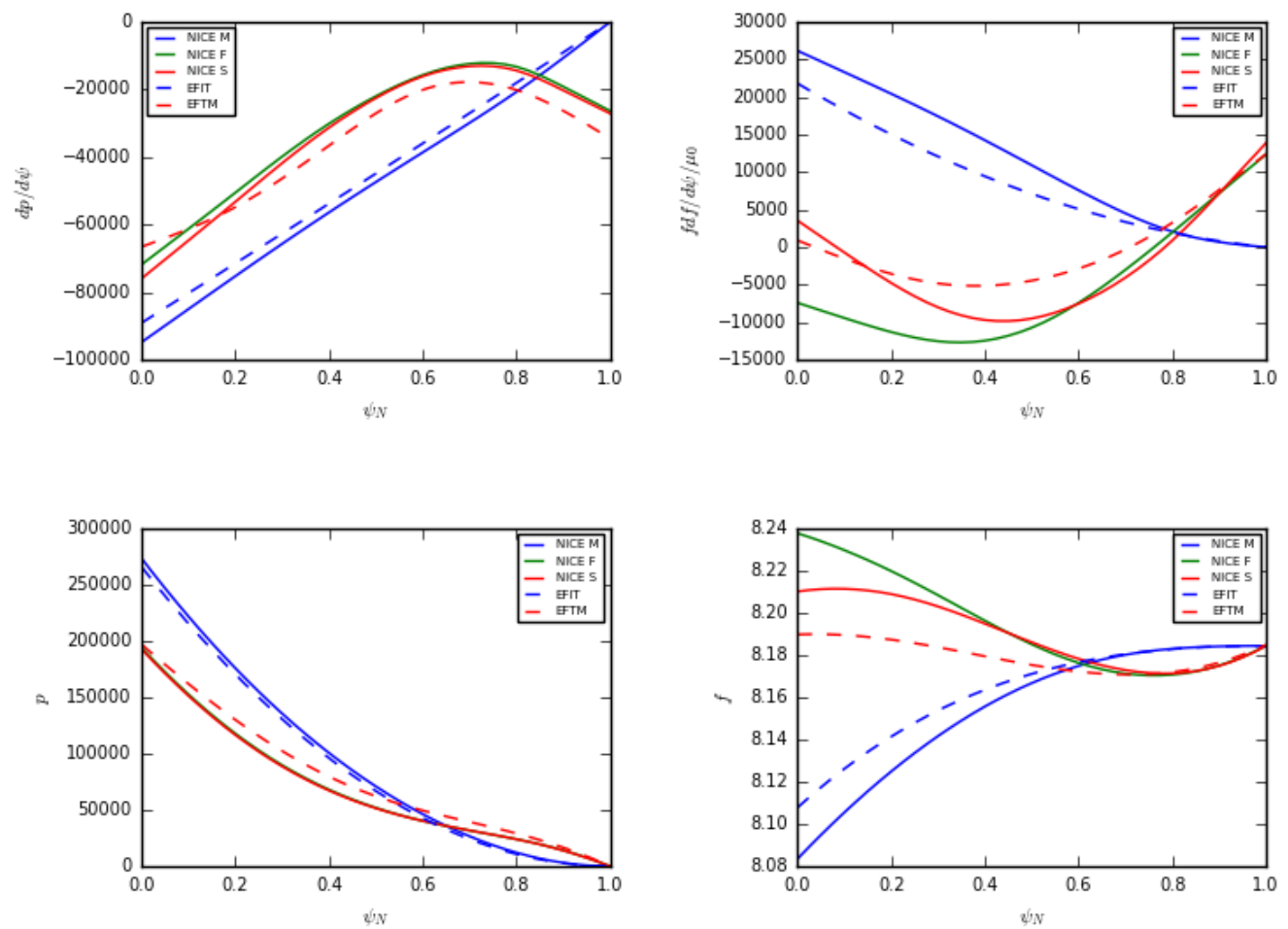

Figure 14: Shot 92398, time 47s. $p^{\prime}, f f^{\prime}$ (first row) and $p, f$ (second row) reconstructed profiles as function of the normalized flux $\psi_{N}$. NICE M (continuous blue), NICE F (continuous green) and NICE S (continuous red), EFIT (dashed blue) and EFTM (dashed red). 

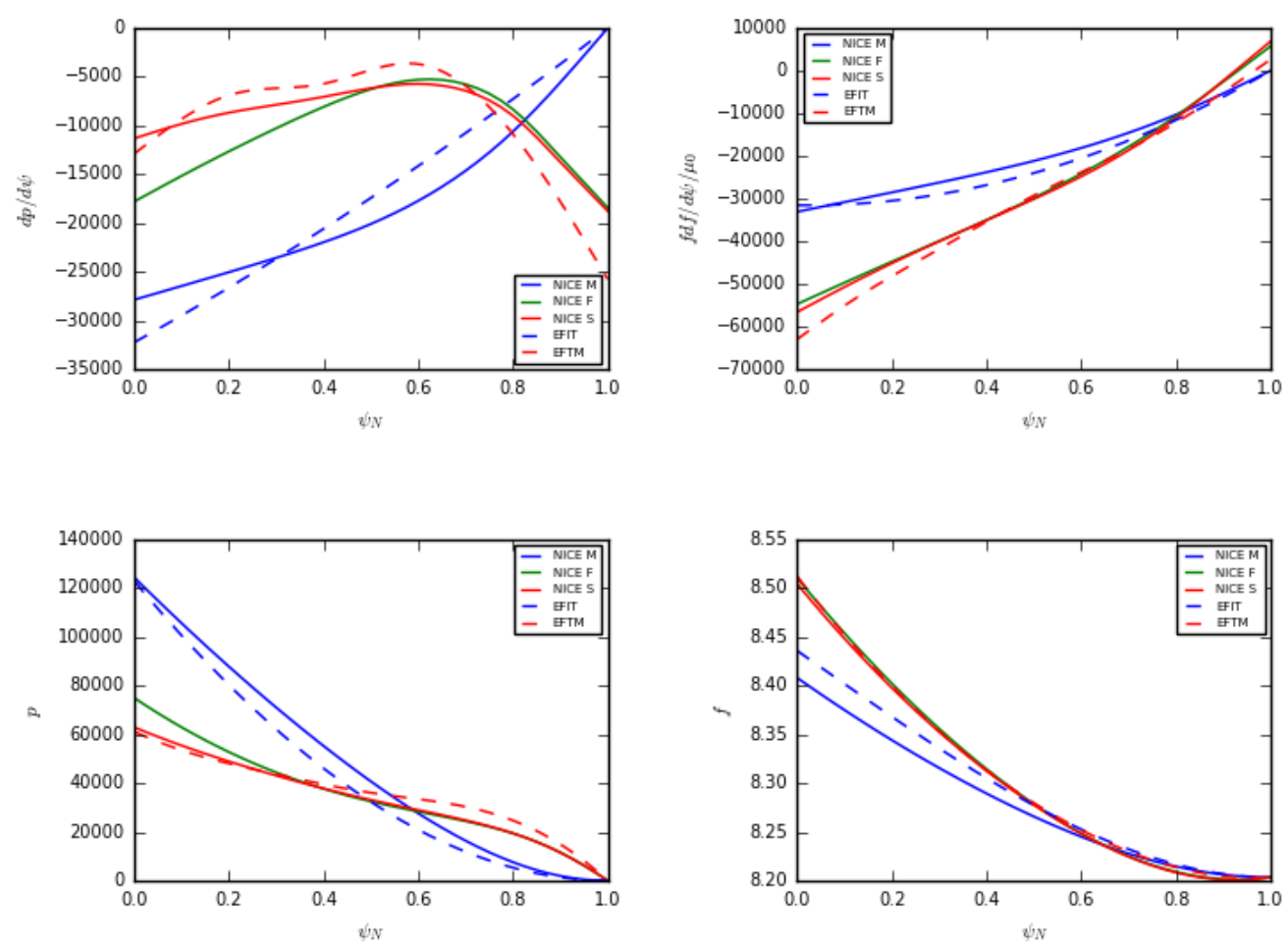

Figure 15: Shot 92436, time 48s. $p^{\prime}, f f^{\prime}$ (first row) and $p, f$ (second row) reconstructed profiles as function of the normalized flux $\psi_{N}$. NICE M (continuous blue), NICE F (continuous green) and NICE S (continuous red), EFIT (dashed blue) and EFTM (dashed red). 

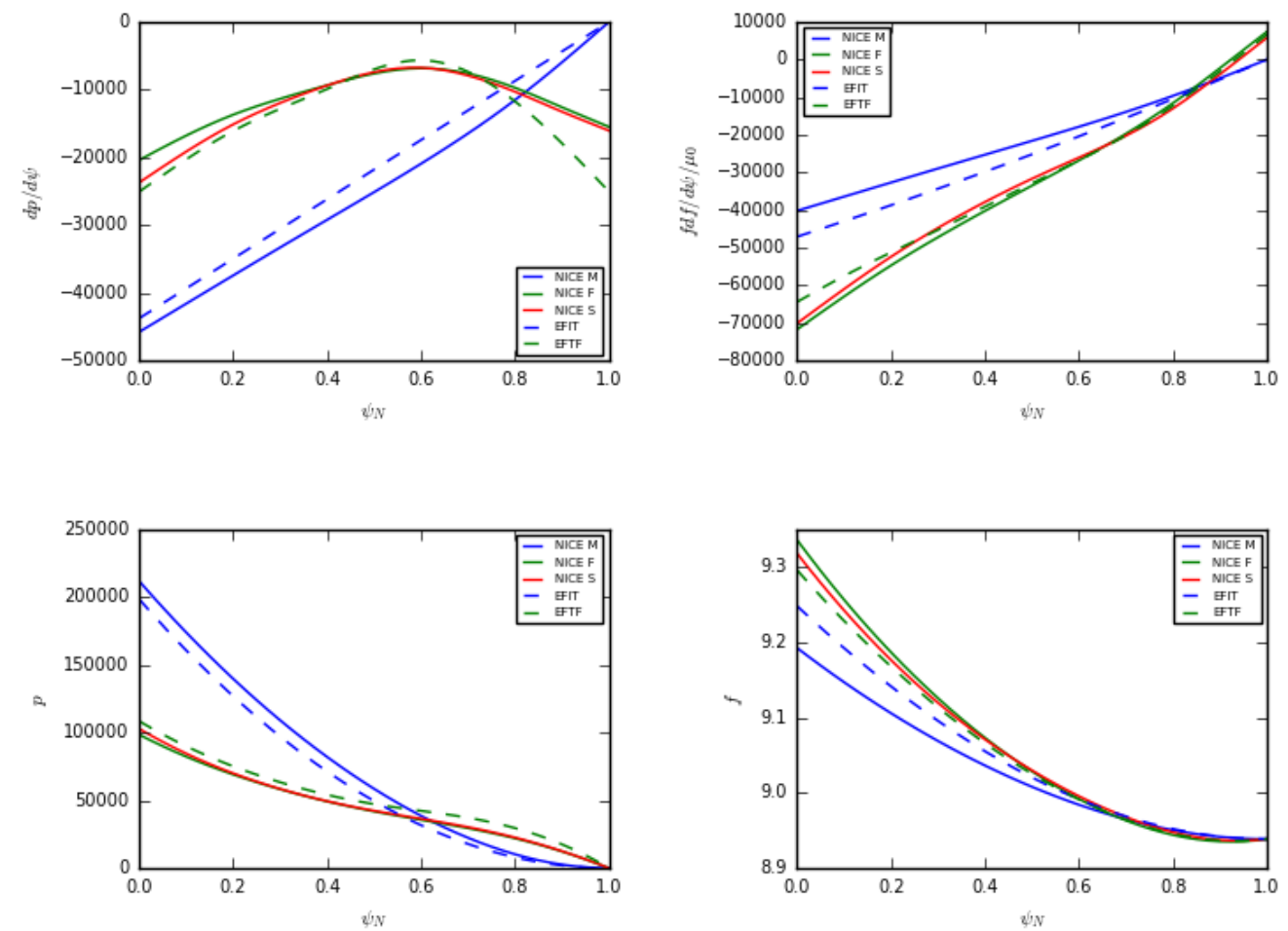

Figure 16: Shot 92441, time 50s. $p^{\prime}, f f^{\prime}$ (first row) and $p, f$ (second row) reconstructed profiles as function of the normalized flux $\psi_{N}$. NICE M (continuous blue), NICE F (continuous green) and NICE S (continuous red), EFIT (dashed blue) and EFTF (dashed green). 


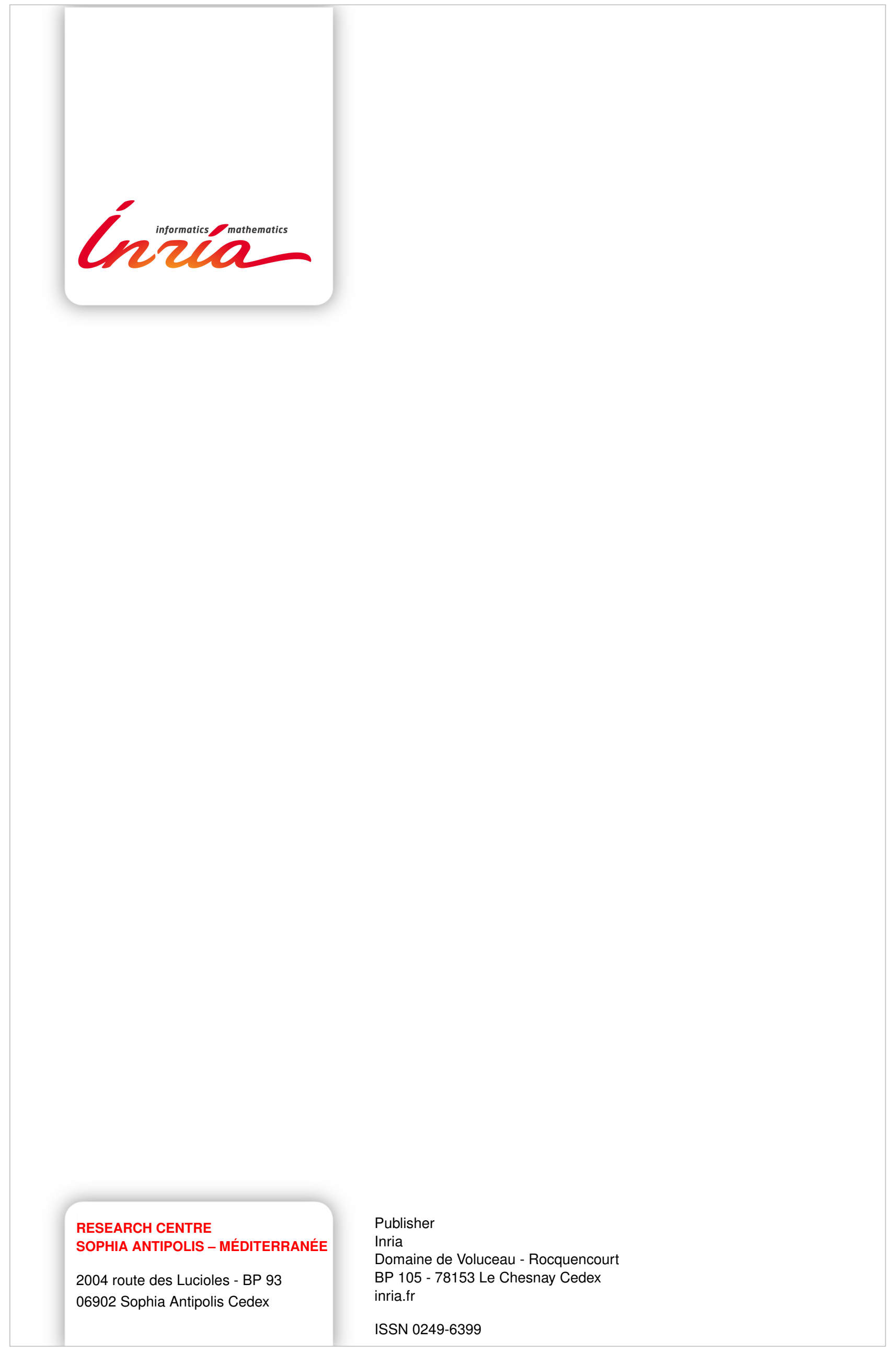

